\title{
Distorted spin-dependent spectral function of an $A=3$ nucleus and semi-inclusive deep-inelastic scattering processes
}

\author{
L. P. Kaptari,,${ }^{1,2,3}$ A. Del Dotto, ${ }^{4}$ E. Pace, ${ }^{5}$ G. Salmè, ${ }^{6}$ and S. Scopetta ${ }^{1}$ \\ ${ }^{1}$ Department of Physics and Geology, University of Perugia, and Istituto Nazionale di Fisica Nucleare, Sezione di Perugia, \\ Via Alessandro Pascoli, Perugia I-06123, Italy \\ ${ }^{2}$ Thomas Jefferson National Accelerator Facility Newport News, Virginia 23606, USA \\ ${ }^{3}$ Bogoliubov Laboratory of Theoretical Physics, JINR, 141980 Dubna, Russia \\ ${ }^{4}$ Department of Physics and Mathematics, University of Rome "Roma Tre" and Istituto Nazionale di Fisica Nucleare, \\ Sezione di "Roma Tre," Via della Vasca Navale 84, 00146 Rome, Italy \\ ${ }^{5}$ Physics Department, University of Rome "Tor Vergata” and Istituto Nazionale di Fisica Nucleare, Sezione di Tor Vergata, \\ Via della Ricerca Scientifica 1, I-00133 Rome, Italy \\ ${ }^{6}$ Istituto Nazionale di Fisica Nucleare, Sezione di Roma, Piazzale Aldo Moro 2, I-00185 Rome, Italy
}

(Received 11 July 2013; revised manuscript received 8 November 2013; published 27 March 2014)

\begin{abstract}
The distorted spin-dependent spectral function of a nucleon inside an $A=3$ nucleus is introduced as a novel tool for investigating the polarized electron scattering off polarized ${ }^{3} \mathrm{He}$ in the semi-inclusive DIS regime (SiDIS), going beyond the standard plane-wave impulse approximation. This distribution function is applied to the study of the spectator $\operatorname{SiDIS},{ }^{3} \mathrm{He}\left(\vec{e}, e^{\prime 2} \mathrm{H}\right) X$, to properly take into account the final-state interaction between the hadronizing quark and the detected deuteron, with the final goal of a more reliable extraction of the polarized parton distribution $g_{1}(x)$ inside a bound proton. Our analysis allows one to single out two well-defined kinematical regions where the experimental asymmetries could yield very interesting information: the region where the final-state effects can be minimized, and therefore the direct access to the parton distributions in the proton is feasible, and the one where the final-state interaction dominates, and the spectator SiDIS reactions can elucidate the mechanism of the quark hadronization itself. The perspectives of extending our approach (i) to the mirror nucleus, ${ }^{3} \mathrm{H}$, for achieving a less model-dependent flavor decomposition and (ii) to the asymmetries measured in the standard SiDIS reactions, $\vec{e}+{ }^{3} \overrightarrow{\mathrm{He}} \rightarrow e^{\prime}+h+X$, with $h$ a detected fast hadron, with the aim of extracting the neutron transversity, are discussed.
\end{abstract}

DOI: 10.1103/PhysRevC.89.035206

\section{INTRODUCTION}

Inclusive deep-inelastic scattering (DIS), i.e., the process $A\left(l, l^{\prime}\right) X$, where a lepton interacts with a hadronic target $A$ and only the scattered lepton is detected, has been the subject of intense experimental investigation in the past decades. Through these processes it has become possible to describe the longitudinal (with respect to the direction of the high energy beam) momentum and helicity distributions of the partons, in both proton (see, e.g., Refs. [1,2]) and nuclear targets (see, e.g., Refs. [3-5]).

Despite of these efforts it is nowadays clear that the answers to a few crucial questions can be hardly found through DIS experiments. Among them, we identify at least three challenging problems, which are related to the subject of this paper: (i) the fully quantitative explanation of the so-called European Muon Collaboration (EMC) effect (i.e., the modification of the nucleon partonic structure owing to the nuclear medium, observed long time ago [6]); (ii) the solution of the so-called "spin crisis," i.e., the the observation that the nucleon spin does not originate from only the spins of its valence quarks, already reported in Ref. [7]; (iii) the measurement of the chiral-odd parton distribution function (PDF) called transversity (see, e.g., Refs. [8,9] for an introduction to this issue), related to the probability distribution to find a transversely polarized quark inside a transversely polarized nucleon, that allows one to complete the leading-twist description of a polarized nucleon, unfortunately not measurable in DIS.
PACS number(s): 13.40.-f, 24.85.+p, 25.10.+s, 25.30.Fj

To clarify the above-mentioned problems, a new direction has been taken. Special efforts on both experimental and theoretical sides have been focused on exclusive and semiinclusive deep-inelastic (SiDIS) measurements, to go beyond the inclusive processes. In the SiDIS reactions, besides the scattered lepton, a hadron is detected in coincidence; see Fig. 1. If the detected hadron is fast, one can expect that it originates from the fragmentation of the active, highly off-mass-shell quark, after absorbing the virtual photon. Hence, the detected hadron brings valuable information about the motion of quarks in the parent nucleon before interacting with the photon, and in particular on their transverse motion. Therefore, through SiDIS reactions, one can access the so-called transverse momentum-dependent (TMD) parton distributions (see, e.g., Refs. [8,9]) (i) establishing a framework where one can develop new tools for gathering a wealth of information on the partonic dynamics, more rich than in the collinear case, and (ii) eventually shedding light on the above-mentioned three issues. Incidentally, beside the main topic represented by TMDs, one should remember that the detected hadron carries also information on the hadronization mechanism itself. The SiDIS cross sections can be parametrized, at leading twist, by six parton distributions; this number reduces to three in DIS and increases to eight once the so-called time-reversal odd TMDs are considered [8]. In view of this, SiDIS reactions play a crucial role for addressing the issue of the chiral-odd transversity, because it is necessary to flip the quark chirality 


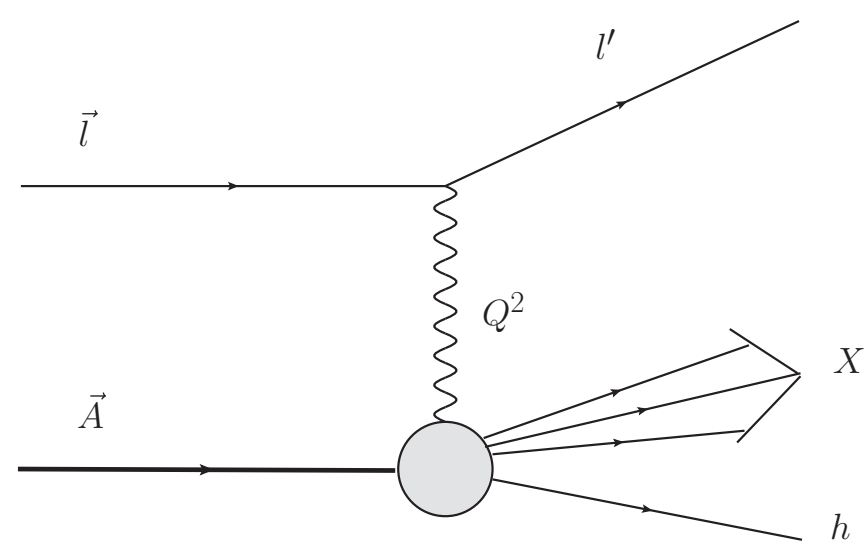

FIG. 1. One-photon exchange diagram for SiDIS processes $\vec{A}\left(\vec{l}, l^{\prime} h\right) X$. The target $A$ can be either longitudinally or transversely polarized.

for observing this TMD. Finally, it should be emphasized that, to experimentally investigate the wide field of TMDs, one should measure cross-section asymmetries, using different combinations of beam and target polarizations (see, e.g., Ref. [10]). Moreover, to make complete the study of TMDs one should achieve a sound flavor decomposition, which can be obtained once neutron data become available. To this end, it should be pointed out that the investigation of the neutron content is highly favored by choosing a polarized ${ }^{3} \mathrm{He}$ as a target, as discussed in detail in what follows.

As is well known, free neutron targets are not available and nuclei have to be used as effective neutron targets. Owing to its peculiar spin structure (see, e.g., Ref. [11]), polarized ${ }^{3} \mathrm{He}$ has been used extensively in DIS studies. In particular, procedures to extract the neutron spin-dependent structure functions from ${ }^{3} \mathrm{He}$ data, taking properly into account the Fermi motion and binding effects, have been proposed and successfully applied [12]. Such a detailed description of the target nucleus was accomplished in plane-wave impulse approximation (PWIA) by using the so-called spin-dependent spectral function, whose diagonal elements yield the probability distribution to find a nucleon with a given momentum, missing energy, and polarization inside the nucleus. It is worth noting that, within PWIA, accurate ${ }^{3} \mathrm{He}$ spin-dependent spectral functions, based on realistic few-body calculations (for both the target nucleus and the spectator pair in the final state), have been built in the past 20 years [13-16]. It is very important to notice that the spin-dependent spectral function contains two contributions: One does not depend upon the polarization of the target nucleus, $\mathbf{S}_{A}$, and the other one does. This is the term that enters the description of the asymmetries of the cross sections we are going to investigate, and for the sake of brevity we call it spin-dependent contribution.

The question of whether similar procedures, based on the PWIA spectral function, can be extended to SiDIS is of great relevance, owing to several experiments that exploit a polarized ${ }^{3}$ He target (see, e.g., Ref. [17]), for investigating the transverse degrees of freedom of the neutron. For instance, wide interest has arisen about the possibility to measure, using transversely polarized ${ }^{3} \mathrm{He}$, azimuthal single-spin asymmetries of the neutron, which are sensitive to (i) the so-called time-reversal odd TMDs (such as the Sivers [18] and Boer-Mulders [19] functions) and (ii) fragmentation functions (such as the Collins function [20]). Indeed, it is the existence of final-state interactions (FSIs) at leading twist that allows for time-reversal odd TMDs [21]. The first measurements of these functions, obtained through SiDIS off transversely polarized proton and deuteron targets, have produced a puzzling experimental scenario [22,23]. Therefore, with the aim of extracting the neutron information to shed some light on the problem, a measurement of SiDIS off transversely polarized ${ }^{3} \mathrm{He}$ has been addressed [24], and an experiment, planned to measure azimuthal asymmetries in the production of leading $\pi^{ \pm}$from transversely polarized ${ }^{3} \mathrm{He}$, has been already completed at Jefferson Lab (JLab), with a beam energy of $6 \mathrm{GeV}$ [25]. Notice that a new experiment has been planned and will be soon performed after completing the 12-GeV upgrade [26].

In view of these experimental efforts, a realistic PWIA analysis of SiDIS off transversely polarized ${ }^{3} \mathrm{He}$ has been presented in Ref. [27]. A spin-dependent spectral function [16], corresponding to the nucleon-nucleon AV18 interaction [28], has been used for a realistic description of the nuclear dynamics and the crucial issue of extracting the neutron information from ${ }^{3} \mathrm{He}$ data has been discussed. It was found that for SiDIS reactions, where both parton distributions and fragmentation functions are involved, one can safely extend a model-independent extraction procedure, based on the realistic evaluation of the proton and neutron polarizations in ${ }^{3} \mathrm{He}$ [12] and widely used in inclusive DIS, given its ability to take into account effectively the momentum and energy distributions of the polarized bound nucleons in ${ }^{3} \mathrm{He}$.

However, in a SiDIS process the effect of FSI cannot be neglected a priori and it needs to be seriously investigated. This is the aim of the present paper, where a distorted spin-dependent spectral function is introduced, by applying a generalized eikonal approximation (GEA), already successfully exploited for describing unpolarized SiDIS off nuclei [29].

Concerning the mechanism of SiDIS off nuclear targets, one can distinguish at least two, rather different, sets of reactions.

(i) The most familiar, standard process, where the fast hadron is detected mostly in the forward direction. This implies that the hadron was produced by the leading quark. Therefore, such reactions can be used to investigate TMDs inside the hit nucleon.

(ii) The spectator SiDIS, in which there is no detection of any produced (fast) hadron, while the remaining, slow $(A-1)$ nucleon system, acting as a spectator of the photon-nucleon interaction, is detected.

It has been shown that spectator SiDIS can be very useful to investigate the unpolarized deep-inelastic structure functions $F_{1,2}(x)$ of a bound nucleon and therefore to clarify the origin of the EMC effect [29-34]. At the same time, this process can provide also useful information on quark hadronization in medium, complementary to the data obtained so far by the analysis of the standard SiDIS process. Notably, including polarization degrees of freedom would provide information on the spin-dependent structure functions $g_{1,2}(x)$ for bound 
nucleons and, ultimately, on the origin of the polarized EMC effect.

From the theoretical point of view, an important observation is that in both processes, the standard and spectator SiDISs, nuclear effects can be described by the same quantity, a distorted spin-dependent spectral function, once FSI are taken into account. With respect to the one governing the DIS reactions, this novel distribution function is a more complicated object because, besides the momentum, energy, and polarization distributions of the nucleons in ${ }^{3} \mathrm{He}$, it includes also effects of FSI between the produced particles. A thorough knowledge of the distorted spin-dependent spectral function would allow one to reliably separate the effects owing to the nuclear structure from the ones involving TMDs, in the experimental cross sections. Because nuclear effects are expected to be similar in both SiDIS reactions, the spectral function and FSI effects is studied by considering, as a first step, the simplest process, i.e., the spectator SiDIS, described by only two structure functions of the nucleon. Then, one can proceed with the more complicated and involved standard SiDIS, focusing on the extraction of quark TMDs inside the neutron, i.e., the needed ingredients for making complete the flavor decomposition.

The aim of our paper is the evaluation of the distorted spin-dependent spectral function of ${ }^{3} \mathrm{He}$, taking into account, through a GEA (see, e.g., Ref. [29]), the FSI between the $(A-1)$ spectator and the quark debris, produced after DIS off an internal nucleon, with given polarization. The application to the spectator SiDIS reaction, with the detection of a deuteron in the final state, represents a first playground of our approach. As already mentioned, besides its pedagogical relevance, spectator SiDIS reactions could provide unique information on the polarized EMC effect and on the hadronization mechanism of the quark debris during its propagation through the nuclear medium, depending upon the kinematical region one chooses. In particular, once a final deuteron is detected, one can extract information on the proton. To play the same game for the neutron, one should consider a polarized ${ }^{3} \mathrm{H}$ target, still detecting a final deuteron. This SiDIS reaction could seem to be out of the present range of experimental possibilities, but we cannot refrain to mention that valuable achievements have been reached in the last decade in dealing with such a difficult target, as demonstrated by the final approval (with scientific rating A) of an experiment dedicated to DIS by a ${ }^{3} \mathrm{H}$ target, at JLab [35]. Finally, it should be pointed out that the generalization to other SiDIS processes is rather straightforward, even if very heavy from the numerical-evaluation side, and it will be presented elsewhere [36]. At the present stage, the needed relativistic description of the whole spectator SiDIS process is restricted to the kinematics only, as discussed in the following sections, but to embed the very successful nonrelativistic phenomenology (developed over the past decades) in a fully Poincaré covariant approach, it is exploited [36] the light-front framework, which originates from the seminal work by Dirac on the forms of relativistic Hamiltonian dynamics [37] (for an extended review, see Ref. [38]). Indeed, a first formal investigation of a light-front spectral function for a $J=1 / 2$ target has been recently presented in Ref. [39], and this quantity is the building block of our Poincaré covariant description of SiDIS reactions.

The paper is organized as follows. In Sec. II we present the basic formalism for the cross section, valid for any SiDIS process and define the main quantities relevant for the calculations within the PWIA framework. In Sec. III, the spectator $\operatorname{SiDIS}$ reaction ${ }^{3} \overrightarrow{\mathrm{He}}\left(\vec{e}, e^{\prime 2} \mathrm{H}\right) X$ is investigated in detail, (i) introducing the distorted spin-dependent spectral function, which represents the main ingredient of our method for implementing FSI effects, through a GEA, and (ii) adopting an effective cross section obtained from a model of hadronization of the quark debris. In Sec. IV, numerical results for the components of the spin-dependent spectral function, both in PWIA and with FSI effects taken into account, are presented. Moreover, asymmetries are also shown in the most favorable kinematics for gathering information on both (i) the structure function $g_{1}(x)$ of a bound nucleon and (ii) the hadronization process. In Sec. V, conclusions are drawn and perspectives presented.

\section{THE CROSS SECTION}

The differential cross section for the generic SiDIS process depicted by a Feynman-like diagram in Fig. 1 can be written $[8,30]$ as

$$
\frac{d \sigma}{d \varphi_{e} d x_{\mathrm{Bj}} d y}=\frac{\alpha_{\mathrm{em}}^{2} m_{N} y}{Q^{4}} L^{\mu \nu}\left(h_{l}\right) W_{\mu \nu}^{\text {s.i. }}\left(S_{A}, Q^{2}, P_{h}\right),
$$

where, for incoming and outcoming electrons, $Q^{2}=$ $-q^{2}=-\left(k-k^{\prime}\right)^{2}=\vec{q}^{2}-v^{2}=4 \mathcal{E E}^{\prime} \sin ^{2}\left(\theta_{e} / 2\right)$ is the fourmomentum transfer (with $\vec{q}=\vec{k}-\vec{k}^{\prime}, v=\mathcal{E}-\mathcal{E}^{\prime}$, and $\theta_{e} \equiv$ $\left.\theta_{\widehat{\vec{k} k^{\prime}}}\right) ; y=v / \mathcal{E}, x_{\mathrm{Bj}}=Q^{2} / 2 m_{N} v$ the Bjorken scaling variable, $m_{N}$ the nucleon mass, $\alpha_{\mathrm{em}}$ the electromagnetic fine structure constant, and $P_{h}$ the detected-hadron four-momentum.

The leptonic tensor $L_{\mu \nu}$ is an exactly calculable quantity in QED. In the ultrarelativistic limit it gets the form

$$
L_{\mu \nu}\left(h_{l}\right)=2\left[k_{\mu} k_{\nu}^{\prime}+k_{\mu}^{\prime} k_{\nu}-\left(k k^{\prime}\right) g_{\mu \nu}+i h_{l} \varepsilon_{\mu \nu \alpha \beta} k^{\alpha} q^{\beta}\right],
$$

where $h_{e}$ is the helicity of the incident electron and the Levi-Civita tensor $\varepsilon_{\mu \nu \alpha \beta}$ is defined as $\varepsilon_{0123}=-1$. The semi inclusive (s.i.) hadronic tensor of the target with polarization four-vector $S_{A}$ and mass $M_{A}^{2}=P_{A}^{2}$ is defined as

$$
\begin{aligned}
W_{\mu \nu}^{\text {s.i. }}\left(S_{A}, Q^{2}, P_{h}\right)= & \frac{1}{4 \pi M_{A}} \sum_{X}\left\langle S_{A}, P_{A}\left|J_{\mu}\right| P_{h}, X\right\rangle \\
& \times\left\langle P_{h}, X\left|J_{\nu}\right| S_{A}, P_{A}\right\rangle(2 \pi)^{4} \\
& \times \delta^{4}\left(P_{A}+q-P_{X}-P_{h}\right) d \tau_{X} \frac{d \mathbf{P}_{h}}{2 E_{h}(2 \pi)^{3}},
\end{aligned}
$$

where the covariant normalization $\left\langle p \mid p^{\prime}\right\rangle=$ $2 E(2 \pi)^{3} \delta\left(\mathbf{p}-\mathbf{p}^{\prime}\right)$ has been assumed and $d \tau_{X}$ indicates the suitable phase-space factor for the undetected hadronic state $X$. It should be pointed out that in Eq. (3) the integration over the phase-space volume of the detected hadron, $h$, does not have to be performed. 


\section{A. PWIA}

Within PWIA, one can relate the nuclear tensor $W_{\mu \nu}^{\text {s.i. }}\left(S_{A}, Q^{2}, P_{h}\right)$ to the one of a single nucleon $w_{\mu \nu}^{\text {s.i. }}\left(S_{N}, Q^{2}, P_{h}\right)$, by using the following standard assumptions [40]: (i) The nuclear current operator $J_{\mu}$ is approximated by a sum of single nucleon free current operators $j_{\mu}^{N}$; (ii) the interaction of the debris originating by the struck nucleon with the fully interacting $(A-1)$ nuclear system is disregarded, as suggested by the kinematics of the processes under investigation [notice that in this case the channel $1+(A-1)$ is the dominant one in the $A$-nucleon final states]; (iii) the coupling of the virtual photon with the spectator $(A-1)$ system is neglected (given the high momentum transfer), (iv) the effect of the boosts is not considered (they will be properly taken into account in a light-front framework elsewhere [36]). Owing to the assumption (ii) above, the complicated final baryon states $\left|P_{h}, X\right\rangle$ in Eq. (3) are approximated by a tensor product of hadronic states, viz.,

$$
\left|P_{h}, X\right\rangle \stackrel{\text { PWIA }}{\approx}\left|P_{A-1}\right\rangle \otimes\left|P_{h}\right\rangle \otimes\left|X^{\prime}\right\rangle,
$$

where $\left|P_{A-1}\right\rangle$ is a short notation for indicating the state of the fully interacting $(A-1)$-nucleon system, which acts merely as a spectator, $\left|X^{\prime}\right\rangle$ the baryonic state, which originates together with $\left|P_{h}\right\rangle$ from the hadronization of the quark which has absorbed the virtual photon, and of the other colored remnants. The normalization of the $\left|P_{A-1}\right\rangle$ states is

$$
\begin{aligned}
& \sum_{\epsilon_{A-1}^{*}} \rho\left(\epsilon_{A-1}^{*}\right) \int \frac{d \mathbf{P}_{A-1}}{2 E_{A-1}(2 \pi)^{3}}\left|\Phi_{\epsilon_{A-1}^{*}}, \mathbf{P}_{A-1}\right\rangle \\
& \quad \times\left\langle\Phi_{\epsilon_{A-1}^{*}}, \mathbf{P}_{A-1}\right|=1,
\end{aligned}
$$

where $P_{N} \equiv\left\{\mathcal{E}_{N}=\sqrt{m_{N}^{2}+\left|\mathbf{P}_{N}\right|^{2}}, \mathbf{P}_{N}\right\}, \Phi_{\epsilon_{A-1}^{*}}$ is the intrinsic part of the $(A-1)$-nucleon state, with eigenvalue $\epsilon_{A-1}^{*}$, and $E_{A-1}=\sqrt{\left(M_{A-1}^{*}\right)^{2}+\left|\mathbf{P}_{A-1}\right|^{2}}$ with $M_{A-1}^{*}=Z_{A-1} m_{p}+(A-$ $\left.1-Z_{A-1}\right) m_{n}+\epsilon_{A-1}^{*}$. The symbol with the sum overlapping the integral indicates that the $(A-1)$ system has both discrete and continuum energy spectra: This corresponds to negative and positive values of the eigenvalue $\epsilon_{A-1}^{*}$, respectively. In Eq. (5), $\rho\left(\epsilon_{A-1}^{*}\right)$ is the proper state density that for $A=3$ in the two-body break-up (2bbu) and three-body break-up (3bbu) reads

$$
\rho_{2 \mathrm{bbu}}=\frac{1}{(2 \pi)^{3}}, \quad \rho_{3 \mathrm{bbu}}=\frac{1}{(2 \pi)^{6}} \frac{m_{N} \sqrt{m_{N} \epsilon_{2}^{*}}}{2} .
$$

By using the assumptions (i)-(iv) and the approximate final state of Eq. (4), one gets an approximation for the matrix elements of the full current in Eq. (3),

$$
\begin{aligned}
\left\langle P_{h}, X\left|J_{v}\right| S_{A}, P_{A}\right\rangle \approx & \sum_{\lambda} \int \frac{d \mathbf{P}_{N}}{2 \mathcal{E}_{N}(2 \pi)^{3}}\left\langle P_{h} ; X^{\prime}\left|j_{1 v}\right| \lambda, P_{N}\right\rangle \\
& \times\left\langle\lambda, P_{N} ; \Phi_{\epsilon_{A-1}^{*}}, \mathbf{P}_{A-1} \mid S_{A}, P_{A}\right\rangle
\end{aligned}
$$

where the notation $\langle A ; B|$ represents the Cartesian product of the two states $\langle A|$ and $\langle B|$. Moreover, a complete set of nucleon plane waves has been inserted, normalized as

$$
\sum_{\lambda} \int \frac{d \mathbf{P}_{N}}{2 \mathcal{E}_{N}(2 \pi)^{3}}\left|\lambda, P_{N}\right\rangle\left\langle\lambda, P_{N}\right|=1,
$$

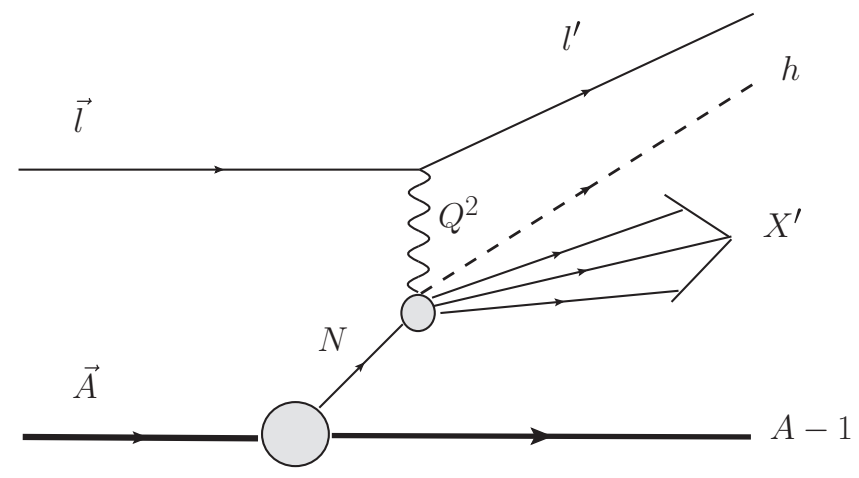

FIG. 2. Diagrammatic representation of SiDIS processes in PWIA. In standard SiDIS reactions, the hadron $h$, originated from the current quark fragmentation, is detected. In spectator SiDIS processes, the $(A-1)$-nucleon system is detected in place of the hadronic state $h$.

between the current operator and the $A$ particle state $\left|P_{A}, S_{A}\right\rangle$. The insertion of the previous completeness leads to the introduction of the notion of spin-dependent spectral function mentioned in the Introduction (see, e.g., Refs. [13-15], for more details). Obviously, the antisymmetry of the identical nucleons is taken care of by the ground-state $\left|P_{A}, S_{A}\right\rangle$. As is well known, the spectral function represents an essential ingredient for describing the nuclear dynamics, but in the presence of FSI one has to properly improve the approach, as discussed in the following sections.

In conclusion, one obtains an expression of the nuclear tensor,

$$
\begin{aligned}
W_{\mu \nu}^{\text {s.i. }}\left(S^{A}, Q^{2}, P_{h}\right)= & \sum_{X^{\prime}, \lambda \lambda^{\prime}} \sum_{N} \int \frac{d E}{(2 \pi)} \mathcal{O}_{\lambda \lambda^{\prime}}^{\hat{\mathbf{s}}_{A}}\left(\mathbf{p}_{N}, E\right) \\
& \times \frac{1}{2 E_{N}}\left\langle\lambda^{\prime}, \tilde{p}_{N}\left|j_{\mu}^{N}\right| P_{h}, X^{\prime}\right\rangle\left\langle P_{h}, X^{\prime}\left|j_{v}^{N}\right| \lambda, \tilde{p}_{N}\right\rangle \\
& \times(2 \pi)^{4} \delta^{4}\left(P_{A}+q-P_{A-1}-P_{h}-P_{X^{\prime}}\right) \\
& \times d \tau_{X^{\prime}} d \mathbf{P}_{A-1} \frac{d \mathbf{P}_{h}}{2 E_{h}(2 \pi)^{3}},
\end{aligned}
$$

where (i) $P_{X^{\prime}}+P_{A-1}$ is in place of $P_{X}$, (ii) the onmass-shell four-momentum of the nucleon is $\tilde{p}_{N} \equiv\left\{E_{N}=\right.$ $\left.\sqrt{m_{N}^{2}+\left|\mathbf{p}_{N}\right|^{2}}, \mathbf{p}_{N}\right\}$, with $\mathbf{p}_{N}=\mathbf{P}_{A}-\mathbf{P}_{A-1}$ the nucleon threemomentum, fixed by the translational invariance of the initial nuclear vertex (cf. Fig. 2), viz.,

$$
\begin{aligned}
\left\langle\Phi_{\epsilon_{A-1}^{*}}, \mathbf{P}_{A-1} \lambda, \tilde{p}_{N} \mid S_{A}, P_{A}\right\rangle= & \sqrt{2 E_{N} 2 E_{A-1} 2 M_{A}}(2 \pi)^{3} \\
& \times \delta\left(\mathbf{P}_{A}-\mathbf{P}_{A-1}-\mathbf{p}_{N}\right) \\
& \times\left\langle\Phi_{\epsilon_{A-1}^{*}}, \mathbf{P}_{A-1} \lambda, \mathbf{p}_{N} \mid S_{A}, \Phi_{A}\right\rangle
\end{aligned}
$$

where $\Phi_{A}$ is the intrinsic wave function of the target nucleus, with mass $M_{A}$ and the factor in front of the $\delta$ function has been chosen to keep the notation of the intrinsic nuclear part as close as possible to the nonrelativistic case, where the plane waves have the normalization given by $\left\langle\mathbf{p} \mid \mathbf{p}^{\prime}\right\rangle=(2 \pi)^{3} \delta\left(\mathbf{p}-\mathbf{p}^{\prime}\right)$. Finally, let us recall that $\left(P_{A}-P_{A-1}\right)^{2} \neq m_{N}^{2}$. It should be 
pointed out that once the assumptions (i)-(iii) described at the begining of the section are introduced, the hadronic tensor in Eq. (9) no longer fulfills the current conservation, and only phenomenological prescriptions can be adopted (see, e.g., Ref. [41] for a discussion of the quasielastic region and Refs. [42-44] for the DIS one within a covariant approach, i.e., with off-mass shell constituents and four-momentum conservation at each interaction vertex where a constituent interacting with the other constituents is involved). Indeed, to have a conserved current one should consider an interactiondependent many-body e.m. current, e.g., the one investigated in Ref. [45] within the light-front approach. In what follows, the antisymmetric part of the hadronic tensor, which can be modeled by using the factor $\epsilon^{\mu \nu \alpha \beta} q_{\alpha}$ (see below) and therefore explicitly fulfills the current conservation, is relevant.

The effects of the nuclear structure in Eq. (9) are encoded in the overlaps $\mathcal{O}_{\lambda^{\prime} \lambda}^{\hat{\mathbf{S}}_{A}}\left(\mathbf{p}_{N}, E\right)$, defined as

$$
\begin{aligned}
\mathcal{O}_{\lambda \lambda^{\prime}}^{\hat{\mathbf{s}}_{A}}\left(\mathbf{p}_{N}, E\right)= & \sum_{\epsilon_{A-1}^{*}} \rho\left(\epsilon_{A-1}^{*}\right)\left\langle\Phi_{\epsilon_{A-1}^{*}}, \lambda, \mathbf{p}_{N} \mid S_{A}, \Phi_{A}\right\rangle \\
& \times\left\langle S_{A}, \Phi_{A} \mid \Phi_{\epsilon_{A-1}^{*}}, \lambda^{\prime}, \mathbf{p}_{N}\right\rangle \\
& \times \delta\left(E+M_{A}-m_{N}-M_{A-1}^{*}-T_{A-1}\right),
\end{aligned}
$$

where $T_{A-1}$ is the kinetic energy of the $A-1$ system. In a nonrelativistic approach, such contribution is disregarded, leading to the identification of $E$ with the usual missing energy, $E=\epsilon_{A-1}^{*}+B_{A}$, with $B_{A}$ the binding energy of the target nucleus. It should be pointed out that $m_{N}-E$ is the energy of a nucleon inside the target nucleus, where the $A-1$ system acts as a spectator. It is important to emphasize that the overlaps are nothing other than the matrix elements of the $2 \otimes 2$ spindependent spectral function of a nucleon inside the nucleus $A$, with polarization $\mathbf{S}_{A}$ [15], the crucial quantity to be introduced in the next section. The diagonal part yields the probability distribution to find a nucleon in the nucleus $A$ with threemomentum $\mathbf{p}_{N}$, missing energy $E$, and spin projection equal to $\lambda$. This entails the following normalization:

$$
\frac{1}{2} \sum_{\lambda} \int d E \int d \mathbf{p}_{N} \mathcal{O}_{\lambda \lambda}^{\hat{\mathbf{s}}_{A}}\left(\mathbf{p}_{N}, E\right)=1 .
$$

In what follows we consider the polarized target in a pure state with the nuclear wave functions having definite spin projections on the spin quantization axis, usually chosen along the polarization vector $\mathbf{S}_{A}$. Accordingly, in the complete set of the nucleon plane waves, the spin projections $\lambda$ and $\lambda^{\prime}$ are defined with respect to this direction. As for the Cartesian coordinates, we adopt the DIS convention; i.e., the $z$ axis is directed along the three-momentum transfer $\mathbf{q}$ and the plane $(x, z)$ is the scattering plane. Moreover, in the DIS limit, the direction of the three-momentum transfer coincides with that of the lepton beam, $\mathbf{q} \| \mathbf{k}_{e}$.

Notice that the s.i. tensor defined by Eq. (9) refers to both kinds of SiDIS. Indeed, the standard SiDIS implies integrations over $d \tau_{X^{\prime}}$ and $d \mathbf{P}_{A-1}$, while for the spectator process the integrations are performed over $d \tau_{X^{\prime}}$ and $d \mathbf{P}_{h} /\left[2 E_{h}(2 \pi)^{3}\right]$, respectively. By inserting Eq. (9) in Eq. (1), the cross section for standard SiDIS, when the hadron $h$ is detected, is obtained as

$$
\begin{aligned}
2 E_{h} \frac{d \sigma\left(h_{l}\right)}{d \varphi_{e} d x_{\mathrm{Bj}} d y d \mathbf{P}_{h}}= & \frac{\alpha_{\mathrm{em}}^{2} y}{2 Q^{4}} L^{\mu \nu}\left(h_{l}\right) \sum_{\lambda \lambda^{\prime}} \sum_{N} \int d \mathbf{p}_{N} \\
& \times \int d E \frac{m_{N}}{E_{N}} w_{\mu \nu}^{\text {s.i. }}\left(p_{N}, P_{h}, \lambda \lambda^{\prime}\right) \\
& \times \mathcal{O}_{\lambda \lambda^{\prime}}^{\hat{\mathbf{S}}_{A}}\left(\mathbf{p}_{N}, E\right),
\end{aligned}
$$

where the integration over $\mathbf{P}_{A-1}$ has been traded off with the one over $\mathbf{p}_{N}=\mathbf{P}_{A}-\mathbf{P}_{A-1}$, and the s.i. nucleon tensor [cf. Eq. (3)] is given by

$$
\begin{aligned}
w_{\mu \nu}^{\text {s.i. }}\left(p_{N}, P_{h}, \lambda^{\prime} \lambda\right)= & \sum_{X^{\prime}}\left\langle\tilde{p}_{N}, \lambda^{\prime}\left|j_{\mu}\right| P_{h}, X^{\prime}\right\rangle\left\langle P_{h}, X^{\prime}\left|j_{v}\right| \tilde{p}_{N}, \lambda\right\rangle \\
& \times \delta^{4}\left(p_{N}+q-P_{h}-P_{X^{\prime}}\right) d \tau_{X^{\prime}},
\end{aligned}
$$

where $p_{N}=P_{A}-P_{A-1} \equiv\left\{m_{N}-E, \mathbf{p}_{N}\right\}$ is such that $p_{N}^{2} \neq$ $m_{N}^{2}=\tilde{p}_{N}^{2}$. It is interesting to recall that within the lightfront description of a Hamiltonian system [37,38] combined with the Bakamjian-Thomas construction of the Poincaré generators [46], the constituents are on their own mass shell, and only the light-front three-momentum is conserved at the interaction vertex where a constituent interacting with the other constituents is involved. This framework represents an alternative to the one adopted to the covariant approaches adopted in Refs. [42-44], and it will be explored elsewhere [36].

The cross section for the spectator SiDIS [when the slow $(A-1)$ system is detected] has the same structure as the one in Eq. (14). However, in this case, the integration over the hadronic variables $P_{h}$ has to be performed and the nucleon tensor is of a pure inclusive DIS nature, viz.,

$$
\begin{aligned}
w_{\mu \nu}^{\mathrm{DIS}}\left(p_{N}, Q^{2}, \lambda^{\prime} \lambda\right)= & \frac{1}{(2 \pi)} \sum_{X^{\prime \prime}}\left\langle\tilde{p}_{N}, \lambda^{\prime}\left|j_{\mu}\right| X^{\prime \prime}\right\rangle\left\langle X^{\prime \prime}\left|j_{\nu}\right| \tilde{p}_{N}, \lambda\right\rangle \\
& \times(2 \pi)^{4} \delta^{4}\left(p_{N}+q-P_{X^{\prime \prime}}\right) d \tau_{X^{\prime \prime}}
\end{aligned}
$$

In Eq. (15), the final state $X^{\prime \prime}$ could be $X^{\prime}+h$, with the notation in Fig. 2, but, obviously, could be any other state accessible from the given initial state. In this case, the cross section becomes

$$
\begin{aligned}
\frac{d \sigma\left(h_{l}\right)}{d \varphi_{e} d x_{\mathrm{Bj}} d y d \mathbf{P}_{A-1}}= & \frac{\alpha_{\mathrm{em}}^{2} y}{2 Q^{4}} L^{\mu \nu}\left(h_{l}\right) \sum_{\lambda \lambda^{\prime}} \sum_{N} \int d E \frac{m_{N}}{E_{N}} w_{\mu \nu}^{\mathrm{DIS}} \\
& \times\left(p_{N}, Q^{2}, \lambda^{\prime} \lambda\right) \mathcal{O}_{\lambda \lambda^{\prime}}^{\hat{\mathbf{S}}_{A}}\left(\mathbf{p}_{N}, E\right) .
\end{aligned}
$$

In conclusion, Eqs. (13) and (16) show that the central quantities for describing SiDIS reactions in PWIA are (i) the overlap integrals $\mathcal{O}_{\lambda \lambda^{\prime}}^{\hat{\mathbf{S}}_{A}}\left(\mathbf{p}_{N}, E\right)$, which contain information on the nuclear-structure effects and (ii) the suitable tensor $w_{\mu \nu}$ of a moving nucleon. In particular, the antisymmetric part of the nucleon tensor is the basic ingredient in the evaluation of proper cross-section asymmetries, which represent the main goal of the experimental investigation of SiDIS reactions.

\section{B. The antisymmetric tensor $w_{\mu v}^{a \mathrm{DIS}}$ of a moving nucleon}

In this paper we focus on the spectator SiDIS and in particular on the asymmetries of the cross sections, obtained 
properly varying the polarization of the involved particles. Therefore, the antisymmetric part of the nucleon tensor $w_{\mu \nu}^{\text {DIS }}$ [cf. Eq. (15)] is the relevant quantity. Following Ref. [15] (see also Ref. [14]), the antisymmetric part of the tensor for a nucleon with a definite polarization $S_{N}$ is given by

$$
w_{\mu \nu}^{a, \text { DIS }}\left(p_{N}, Q^{2}, \lambda^{\prime} \lambda\right)=\left\langle\lambda^{\prime}\left|\hat{w}_{\mu \nu}^{a N}\right| \lambda\right\rangle,
$$

where the operator $\hat{w}_{\mu \nu}^{a N}\left(p_{N}, Q^{2}, S_{N}\right)$ in Eq. (17) can be written as $[12,14,15,43,44,47]$

$$
\begin{aligned}
\hat{w}_{\mu \nu}^{a N}\left(p_{N}, Q^{2}, S_{N}\right) & \\
= & i \varepsilon_{\mu \nu \alpha \beta} q^{\alpha}\left\{m_{N} \hat{S}_{N}^{\beta} G_{1}^{N}\left(Q^{2}, p_{N} q\right)+\frac{G_{2}^{N}\left(Q^{2}, p_{N} \cdot q\right)}{m_{N}}\right. \\
& \left.\times\left[\left(p_{N} q\right) \hat{S}_{N}^{\beta}-\left(S_{N} q\right) p_{N}^{\beta}\right]\right\}
\end{aligned}
$$

where the two scalar functions $G_{1,2}$ are the polarized DIS structure functions and the quantity $\hat{S}_{N}$ is the four-vector polarization operator acting in the $2 \times 2$ spin space. It is defined as

$$
\hat{S}_{N}^{\beta}= \begin{cases}\frac{\left(\sigma \mathbf{p}_{N}\right)}{m_{N}}, & \beta=0, \\ \sigma+\mathbf{p}_{N} \frac{\left(\sigma \mathbf{p}_{N}\right)}{m_{N}\left(E_{N}+m_{N}\right)}, & \beta=1,2,3,\end{cases}
$$

with $\sigma$ the usual Pauli matrices. As previously mentioned, the antisymmetric part of the nucleon tensor we are adopting is explicitly current conserved and, in turn, the hadronic tensor is as well. In the present calculation, as in the relativistic Hamiltonian dynamics framework, e.g., the one adopted in Ref. [48], the dependence on $p_{N}^{2} \neq m_{N}^{2}$ of the nucleon structure functions $G_{1,2}$ is neglected. In a forthcoming analysis of ours [36], a light-front approach combined with the Mandelstam formula for the current matrix elements will be applied to shed light on the above issue.

In standard SiDIS, the analogs of the operator $\hat{w}_{\mu \nu}^{a N}\left(p_{N}, Q^{2}, S_{N}\right)$ becomes a more complicated object, because, within the quark parton model, it can be expressed as a convolution of the TMDs with different quark fragmentation functions (see, e.g., Ref. [8]).

\section{SPECTATOR SIDIS BY A POLARIZED ${ }^{3}$ He TARGET}

As shown in Eqs. (13) and (16), the nuclear effects in both SiDIS reactions are governed by the overlap integrals $\mathcal{O}_{\lambda \lambda^{\prime}}^{\hat{\mathbf{S}}_{A}}\left(\mathbf{p}_{N}, E\right)$. In this paper we focus mostly on the investigation of nuclear effects, and therefore we consider the spectator SiDIS, which has a nucleon tensor, $w_{\mu \nu}^{\text {DIS }}$, with fewer uncertainties in the parton structure (calculations of the asymmetries for the standard SiDIS process using different TMDs and fragmentation functions in the planned JLAB kinematics will be reported elsewhere [36]). In particular, we consider the case of a polarized ${ }^{3} \mathrm{He}$ target, but as mentioned in the Introduction, one can repeat the same considerations for ${ }^{3} \mathrm{H}$, modulo the Coulomb effects. For the sake of simplicity, we choose the simplest channel, namely the one with a deuteron in the final state; this means that one can address the proton structure functions inside the ${ }^{3} \mathrm{He}$ target, while in the mirror nucleus one can study the neutron structure functions. Generalization to the case when the detected system is a two-particle state in the continuum is straightforward, but more involved, In particular, we analyze polarized SiDIS with a longitudinal setup; i.e., the polarization of the initial electron and the target nucleus are defined with respect to the direction of the momentum transfer q. If the detected unpolarized $(A-1)$-nucleon system is a deuteron, $\epsilon_{A-1}^{*}=-B_{D}$, then the nucleon missing energy is just the 2 bbu threshold energy of ${ }^{3} \mathrm{He}$, i.e., $E_{2 \mathrm{bbu}}=B_{{ }^{3} \mathrm{He}}-B_{D}$. For the final state we have chosen, the cross section reads

$$
\frac{d \sigma^{\hat{\mathbf{s}}_{A}}\left(h_{e}\right)}{d \varphi_{e} d x_{\mathrm{Bj}} d y d \mathbf{P}_{D}}=\frac{\alpha_{\mathrm{em}}^{2} m_{N} y}{Q^{4}} L^{\mu \nu}\left(h_{e}\right) W_{\mu \nu}^{\text {s.i. }}\left(S^{A}, Q^{2}, P_{D}\right) .
$$

In the asymmetry we are going to investigate, given by

$$
\frac{\Delta \sigma^{\hat{\mathbf{S}}_{A}}}{d \varphi_{e} d x_{\mathrm{Bj}} d y d \mathbf{P}_{D}} \equiv \frac{d \sigma^{\hat{\mathbf{S}}_{A}}\left(h_{e}=1\right)-d \sigma^{\hat{\mathbf{S}}_{A}}\left(h_{e}=-1\right)}{d \varphi_{e} d x_{\mathrm{Bj}} d y d \mathbf{P}_{D}},
$$

only the antisymmetric part of both leptonic and nuclear tensors are involved. In particular, the antisymmetric part of

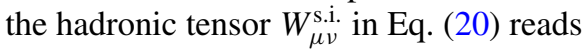

$$
\begin{aligned}
& W_{\mu \nu}^{a, \text { s.i. }}\left(S^{A}, Q^{2}, P_{D}\right) \\
& \quad=\sum_{\lambda \lambda^{\prime}} \frac{1}{2 E_{N}}\left\langle\lambda^{\prime}\left|\hat{w}_{\mu \nu}^{a N}\left(p_{N}, Q^{2}, S_{N}\right)\right| \lambda\right\rangle \mathcal{O}_{\lambda \lambda^{\prime}}^{\hat{\mathrm{S}}_{A}}\left(p_{N}, E_{2 \mathrm{bbu}}\right),
\end{aligned}
$$

with the nucleon DIS tensor $w_{\mu \nu}^{\mathrm{DIS}}\left(p_{N}, Q^{2}, \lambda, \lambda^{\prime}\right)$ given formally in Eqs. (15) and then explicitly in Eqs. (17) and (18). In the DIS limit, the nucleon structure function $G_{2}^{N}$ yields, at the leading twist, a vanishing contribution to the measured cross section. Therefore, in all the following calculations, contributions from $G_{2}^{N}$ are neglected. Then, the antisymmetric part of the nucleon tensor becomes

$$
\begin{aligned}
\left\langle\lambda^{\prime}\left|\hat{w}_{\mu \nu}^{a N}\right| \lambda\right\rangle= & i G_{1}^{N}\left(Q^{2}, p_{N} q\right) \varepsilon_{\mu \nu \alpha \beta} m_{N} q^{\alpha} \\
& \times \sum_{\kappa}(-1)^{\kappa}\left\langle\lambda^{\prime}\left|\sigma_{-\kappa}\right| \lambda\right\rangle \operatorname{Tr}\left(\frac{1}{2} \sigma_{\kappa} \hat{S}_{N}^{\beta}\right) \\
= & -i \sqrt{3} G_{1}^{N}\left(Q^{2}, p_{N} q\right) \varepsilon_{\mu \nu \alpha \beta} m_{N} q^{\alpha} \\
& \times \sum_{\kappa}(-1)^{\kappa}\left\langle 1-\kappa \frac{1}{2} \lambda \mid \frac{1}{2} \lambda^{\prime}\right\rangle \mathcal{B}_{\kappa}^{\beta},
\end{aligned}
$$

with

$$
\mathcal{B}_{\kappa}^{\beta} \equiv \operatorname{Tr}\left(\frac{1}{2} \sigma_{\kappa} \hat{S}_{N}^{\beta}\right) .
$$

Notice that Eq. (24) defines a "double" vector with double indices: the index $\kappa=0, \pm 1$, labels three four-vectors, with Lorentz index $\beta$. The latter has to be contracted with the corresponding index in the Levi-Civita tensor $\varepsilon_{\mu \nu \alpha \beta}$; see Eq. (18). The Cartesian components of $\mathcal{B}^{\beta}$ are given by (a mixed notation is adopted, but it is self-explanatory)

$$
\mathcal{B}_{i}^{\beta}= \begin{cases}\frac{\left(\mathbf{p}_{N}\right)_{i}}{m_{N}}, & \beta=0, \\ \delta_{\beta i}+\left(\mathbf{p}_{N}\right)^{\beta} \frac{\left(\mathbf{p}_{N}\right)_{i}}{m_{N}\left(E_{N}+m_{N}\right)}, & \beta=1,2,3 .\end{cases}
$$


By placing Eq. (23) into Eq. (22), one can write the nuclear tensor as follows

$$
\begin{aligned}
W_{\mu \nu}^{a, \text { s.i. }}\left(S^{A}, Q^{2}, P_{D}\right)= & i \frac{1}{2} G_{1}^{N}\left(Q^{2}, p_{N} q\right) \varepsilon_{\mu \nu \alpha \beta} \frac{m_{N}}{E_{N}} q^{\alpha} \\
& \times \sum_{\lambda \lambda^{\prime}} \sum_{\kappa}(-1)^{\kappa}\left[-\sqrt{3}\left|1-\kappa \frac{1}{2} \lambda\right| \frac{1}{2} \lambda^{\prime}\right\rangle \\
& \left.\times \mathcal{O}_{\lambda \lambda^{\prime}}^{\hat{\mathbf{s}}_{A}}\left(\mathbf{p}_{N}, E_{2 \mathrm{bbu}}\right)\right] \mathcal{B}_{\kappa}^{\beta} .
\end{aligned}
$$

It can be seen that the dependence upon the index $\kappa$ leads to a scalar product of two vectors, viz.,

$$
\left(\mathcal{P}^{\hat{\mathbf{S}}_{A}} \cdot \mathcal{B}^{\beta}\right) \equiv \sum_{\kappa}(-1)^{\kappa} \mathcal{P}_{-\kappa}^{\hat{\mathbf{S}}_{A}} \mathcal{B}_{\kappa}^{\beta}
$$

where

$$
\mathcal{P}_{\kappa}^{\hat{\mathbf{S}}_{A}}\left(\mathbf{p}_{N}, E_{2 \mathrm{bbu}}\right) \equiv-\sqrt{3} \sum_{\lambda \lambda^{\prime}}\left\langle 1-\kappa \frac{1}{2} \lambda \mid \frac{1}{2} \lambda^{\prime}\right\rangle \mathcal{O}_{\lambda \lambda^{\prime}}^{\hat{\mathbf{s}}_{A}}\left(\mathbf{p}_{N}, E_{2 \mathrm{bbu}}\right)
$$

are the spherical components of the vector $\mathcal{P}^{\hat{\mathbf{S}}_{A}}$, which represents the contribution to the spin-dependent spectral function from the polarization of the target nucleus (see Ref. [15] for details) in a pure state with polarization $\mathbf{S}_{A}$ [we reiterate that in Eq. (28) the spin quantization is along the nuclear polarization $\mathbf{S}_{A}$ ]. Then, the antisymmetric part of the nuclear tensor reads

$$
W_{\mu \nu}^{a, \text { s.i. }}\left(S^{A}, Q^{2}, P_{D}\right)=i \frac{1}{2} G_{1}^{N}\left(Q^{2}, p_{N} q\right) \varepsilon_{\mu \nu \alpha \beta} \frac{m_{N}}{E_{N}} q^{\alpha}\left(\mathcal{P}^{\hat{\mathbf{S}}_{A}} \cdot \mathcal{B}^{\beta}\right)
$$

For further purposes, let us write more explicitly the components of $\mathcal{P}^{\hat{\mathbf{S}}_{A}}$ in both spherical and Cartesian coordinates. By using the spherical versors, one has

$$
\mathcal{P}^{\hat{\mathbf{S}}_{A}}=\mathcal{P}_{\|}^{\hat{\mathbf{S}}_{A}} \mathbf{e}_{0}+\mathcal{P}_{1 \perp}^{\hat{\mathbf{S}}_{A}} \mathbf{e}_{+}+\mathcal{P}_{2 \perp}^{\hat{\mathbf{S}}_{A}} \mathbf{e}_{-}
$$

where $\mathbf{e}_{0} \| \mathbf{S}_{A}$ (see, also Refs. [12,15]) and

$$
\mathbf{e}_{0}=\left(\begin{array}{l}
0 \\
0 \\
1
\end{array}\right), \quad \mathbf{e}_{+}=-\frac{1}{\sqrt{2}}\left(\begin{array}{l}
1 \\
i \\
0
\end{array}\right), \quad \mathbf{e}_{-}=\frac{1}{\sqrt{2}}\left(\begin{array}{c}
1 \\
-i \\
0
\end{array}\right),
$$

or, in terms of Cartesian versors,

$$
\mathcal{P}^{\hat{\mathbf{S}}_{A}}=\mathcal{P}_{x}^{\hat{\mathbf{S}}_{A}} \mathbf{e}_{x}+\mathcal{P}_{y}^{\hat{\mathbf{S}}_{A}} \mathbf{e}_{y}+\mathcal{P}_{z}^{\hat{\mathbf{S}}_{A}} \mathbf{e}_{z} .
$$

Usually, the DIS kinematics is defined in a coordinate system with the $z$ axis along the three-momentum transfer $\mathbf{q}$, whereas the quantization direction to determine the particle polarizations is along the beam direction $\mathbf{k}_{\mathbf{e}}$. In the Bjorken limit $\mathbf{q} \simeq \mathbf{k}_{e}$ and the two directions coincide. This remark will become helpful once FSIs are introduced. The $x$ axis is then chosen to be either in the scattering or in the reaction plane; however, $\mathbf{e}_{y}=\left[\mathbf{e}_{z} \times \mathbf{e}_{x}\right]$.

In terms of the overlap integrals, Eq. (11), the components of $\mathcal{P}^{\hat{\mathbf{S}}_{A}}$ are expressed in spherical basis by

$$
\begin{aligned}
& \mathcal{P}_{\|}^{\hat{\mathbf{S}}_{A}}=\mathcal{O}_{\frac{1}{2} \frac{1}{2}}^{\hat{\mathbf{S}}_{A}}-\mathcal{O}_{-\frac{1}{2}-\frac{1}{2}}^{\hat{\mathbf{S}}_{A}}, \quad \mathcal{P}_{1 \perp}^{\hat{\mathbf{S}}_{A}}=-\sqrt{2} \mathcal{O}_{\frac{1}{2}-\frac{1}{2}}^{\hat{\mathbf{S}}_{A}}, \\
& \mathcal{P}_{2 \perp}^{\hat{\mathbf{S}}_{A}}=\sqrt{2} \mathcal{O}_{-\frac{1}{2} \frac{1}{2}}^{\hat{\mathbf{S}}_{A}},
\end{aligned}
$$

and in Cartesian basis by

$$
\mathcal{P}_{z}^{\hat{\mathbf{S}}_{A}}=\mathcal{P}_{\|}^{\hat{\mathbf{S}}_{A}}, \quad \mathcal{P}_{x}^{\hat{\mathbf{S}}_{A}}=2 \Re \mathcal{O}_{\frac{1}{2}-\frac{1}{2}}^{\hat{\mathbf{S}}_{A}}, \quad \mathcal{P}_{y}^{\hat{\mathbf{S}}_{A}}=-2 \mathfrak{I} \mathcal{O}_{\frac{1}{2}-\frac{1}{2}}^{\hat{\mathbf{S}}_{A}},
$$

It should be noted that, because in Eq. (30) the last two terms are mutually complex conjugated, one has only only two independent components, e.g., $\mathcal{P}_{\|}^{\hat{\mathbf{S}}_{A}}$ and $\mathcal{P}_{1 \perp}^{\hat{\mathbf{S}}_{A}}$. This is a consequence of the fact that in the considered reaction one has at one's disposal only two vectors, $\mathbf{S}_{A}$ and $\mathbf{p}_{N}$, from which a pseudovector $\mathcal{P}^{\hat{\mathbf{S}}_{A}}$ can be constructed [15,39], viz.,

$$
\mathcal{P}^{\hat{\mathbf{S}}_{A}}\left(\mathbf{p}_{N}, E\right)=\mathbf{S}_{A} B_{1}\left(\left|\mathbf{p}_{N}\right|, E\right)+\hat{\mathbf{p}}_{N}\left(\hat{\mathbf{p}}_{N} \cdot \mathbf{S}_{A}\right) B_{2}\left(\left|\mathbf{p}_{N}\right|, E\right),
$$

where $B_{1}\left(\left|\mathbf{p}_{N}\right|, E\right)$ and $B_{2}\left(\left|\mathbf{p}_{N}\right|, E\right)$ are scalar functions to be constructed from the overlaps. By using Eqs. (33) and some algebra, it is easily seen that Eqs. (30) and (35) become equivalent. It should be emphasized that, in the presence of FSI, the spin-dependent spectral function additionally depends upon the direction of the momentum transfer $\mathbf{q}$, so that the simple form given in Eq. (35) no longer holds.

Let us analyze in more detail the 2 bbu contribution to the spin-dependent spectral function of an $A=3$ nucleus, within the PWIA framework. In the actual calculations, both the ${ }^{3} \mathrm{He}$ (target) wave function and the deuteron one correspond to exact solutions of the Schrödinger equation with the AV18 nucleon-nucleon potential [28]. In particular, for ${ }^{3} \mathrm{He}$, the wave function of Ref. [49], but without Coulomb effects, has been adopted; namely, it can be applied for describing also ${ }^{3} \mathrm{H}$.

The overlaps $\mathcal{O}_{\lambda \lambda^{\prime}}^{\hat{\mathbf{S}}_{A}}$ in Eqs. (9) and (11) are explicitly written as

$$
\begin{aligned}
\mathcal{O}_{\lambda \lambda^{\prime}}^{\hat{\mathbf{s}}_{A}}\left(\mathbf{p}_{N}, E_{2 \mathrm{bbu}}\right)= & \sum_{M_{D}}\left[\sum_{\{\alpha, \tilde{\alpha}\}}\left\langle X M_{X} L_{\rho} M_{\rho} \mid \frac{1}{2} M_{A}\right\rangle\left\langle\tilde{X} \tilde{M}_{X} \tilde{L}_{\rho} \tilde{M}_{\rho} \mid \frac{1}{2} M_{A}\right\rangle\left\langle 1 M_{D} \frac{1}{2} \lambda \mid X M_{X}\right\rangle\right. \\
& \left.\times\left\langle 1 M_{D} \frac{1}{2} \lambda^{\prime} \mid \tilde{X} \tilde{M}_{X}\right\rangle(4 \pi)^{2} i^{L_{\rho}}(-i)^{\tilde{L}_{\rho}} \mathrm{Y}_{L_{\rho} M_{\rho}}\left(\hat{\mathbf{p}}_{N}\right) \mathrm{Y}^{*} \tilde{L}_{\rho} \tilde{M}_{\rho}\left(\hat{\mathbf{p}}_{N}\right) O_{\alpha}\left(\left|\mathbf{p}_{N}\right|, E_{2 \mathrm{bbu}}\right) O_{\tilde{\alpha}}\left(\left|\mathbf{p}_{N}\right|, E_{2 \mathrm{bbu}}\right)\right],
\end{aligned}
$$


where the radial overlaps $O_{\alpha}\left(\left|\mathbf{p}_{N}\right|\right)$ are given by

$$
\begin{aligned}
& O_{\alpha}\left(\left|\mathbf{p}_{N}\right|, E_{2 \mathrm{bbu}}\right) \\
& \quad=\int d \rho \rho^{2} \int d r_{23} r_{23} j_{L_{\rho}}\left(\rho\left|\mathbf{p}_{N}\right|\right) R_{\alpha}\left(r_{23}, \rho\right) \Psi_{L_{D}}\left(r_{23}\right),
\end{aligned}
$$

with $\boldsymbol{\rho}$ and $\mathbf{r}_{23}$ the two Jacobi coordinates: $\mathbf{r}_{23}=\mathbf{r}_{2}-\mathbf{r}_{3}$ and $\rho=\mathbf{r}_{1}-\left(\mathbf{r}_{2}+\mathbf{r}_{3}\right) / 2$. In Eq. (36), $\{\alpha\}$ denotes the quantum numbers of a "deuteronlike" configuration, i.e., $L_{\rho}, X, j_{23}=1, L_{23}=L_{D}=0,2$, with the corresponding projections $M_{\rho}, M_{X}, M_{D}$ (see below). Eventually, $R_{\alpha}\left(r_{23}, \rho\right)$ and $\Psi_{L_{D}}\left(r_{23}\right)$ describe the target and deuteron radial wave functions, respectively, viz.,

$$
\begin{aligned}
\left\langle\sigma_{1}, \sigma_{2}, \sigma_{3} ; T_{23}, \tau_{23}, \tau ; \boldsymbol{\rho}, \mathbf{r}_{23} \mid{ }^{3} \mathrm{He} ; \frac{1}{2} M_{A} ; \frac{1}{2} T_{z}\right\rangle \\
=\left\langle T_{23} \tau_{23} \frac{1}{2} \tau \mid \frac{1}{2} T_{z}\right\rangle \sum_{L_{\rho} M_{\rho}} \sum_{X M_{X}} \sum_{j_{23} m_{23}} \\
\quad \times\left\langle X M_{X} L_{\rho} M_{\rho} \mid \frac{1}{2} M_{A}\right\rangle\left\langle j_{23} m_{23} \frac{1}{2} \sigma_{1} \mid X M_{X}\right\rangle \\
\quad \times \sum_{S_{23} m_{S_{23}}} \sum_{L_{23} M_{23}}\left\langle\frac{1}{2} \sigma_{2} \frac{1}{2} \sigma_{3} \mid S_{23} m_{S_{23}}\right\rangle \\
\quad \times\left\langle L_{23} M_{23} S_{23} m_{S_{23}} \mid j_{23} m_{23}\right\rangle \\
\quad \times Y_{L_{23} M_{23}\left(\hat{\mathbf{r}}_{23}\right) Y_{L_{\rho} M_{\rho}}(\hat{\boldsymbol{\rho}}) \phi_{L_{\rho} X}^{j_{23} L_{23} S_{23}}\left(r_{23}, \rho\right),}
\end{aligned}
$$

with $\phi_{L_{\rho} X}^{j_{23} L_{23} S_{23}}\left(r_{23}, \rho\right) \equiv R_{\alpha}\left(r_{23}, \rho\right)$, and

$$
\left\langle\mathbf{r}_{23}, \mid D ; 1 M_{D}\right\rangle=\frac{\Psi_{0_{D}}\left(r_{23}\right)}{r_{23}} \mathcal{Y}_{011}^{M_{D}}\left(\hat{\mathbf{r}}_{23}\right)+\frac{\Psi_{2_{D}}\left(r_{23}\right)}{r_{23}} \mathcal{Y}_{211}^{M_{D}}\left(\hat{\mathbf{r}}_{23}\right) .
$$

In Eq. (36), the $\phi$ dependence of the overlaps, and in turn of $\mathcal{P}^{\hat{\mathrm{S}}_{A}}$ [cf. Eq. (34)] is entirely governed by the difference $M_{\rho}-\tilde{M}_{\rho}$, which does not depend upon the internal summation, namely, $M_{\rho}-\tilde{M}_{\rho}=\lambda^{\prime}-\lambda$. This implies, according to Eq. (33), that the parallel component $\mathcal{P}_{\|}\left(\mathbf{p}_{N}, E\right)$ does not depend upon $\phi$, while the perpendicular ones, $\mathcal{P}_{1 \perp}\left(\mathbf{p}_{N}, E\right)$ and $\mathcal{P}_{2 \perp}\left(\mathbf{p}_{N}, E\right)$, have a functional dependence given by $\exp (\mp i \phi)$, respectively (see Ref. [12]).

\section{Final-state interaction effects}

Let us now consider the effects of FSI. They are attributable to (i) the propagation of the nucleon debris formed after the $\gamma^{*}$ absorption by a target quark, followed by its hadronization and (ii) the interactions of the produced hadrons with the $(A-1)$ spectator system, as schematically depicted in Fig. 3. Indeed, the calculation of such FSI effects from first principles represents a very complicated many-body problem, so that proper model approaches have to be developed. To this end, one is guided by the observation that in the DIS kinematics we are considering: (i) the momentum of the spectator nucleus $\left|\mathbf{P}_{A-1}\right|$ is small; (ii) the large momentum transfer $|\mathbf{q}|$ leads to a very large relative momentum between the debris (with momentum $\mathbf{p}_{X}$ ) and the nucleon $i$ (with momentum $\mathbf{k}_{i}$ ) inside the $(A-1)$ system, i.e., $\left|\left(\mathbf{p}_{X}-\mathbf{k}_{i}\right)\right| \simeq|\mathbf{q}| \gg\left|\mathbf{k}_{i}\right|$ (remember that the distribution of $\left|\mathbf{k}_{i}\right|$ is driven by the target wave

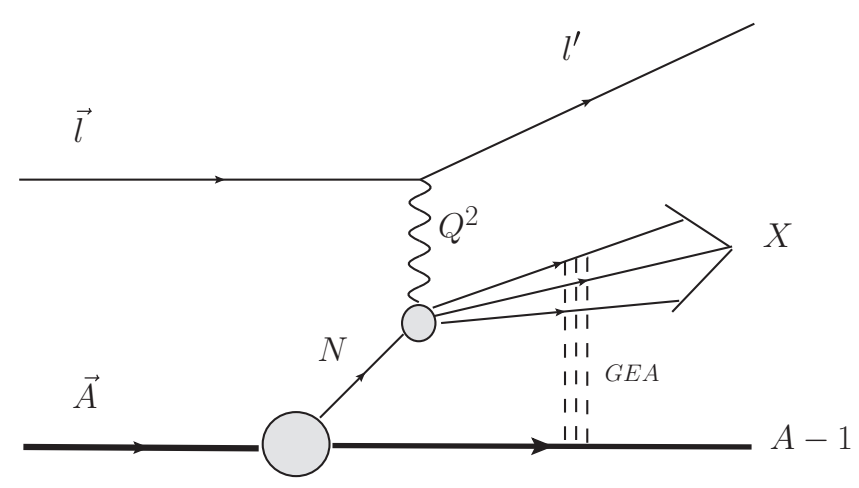

FIG. 3. A diagrammatic illustration of FSI in spectator SiDIS. The rescattering processes between the quark debris and the nucleons inside the $(A-1)$ system are treated within a GEA [29].

function); (iii) the momentum transfer in the rescattering processes, i.e., when the debris interacts with the nucleons inside the $(A-1)$ system, has the typical magnitude of the high-energy elastic $N N$ scattering, i.e., much smaller than the incident momentum $\mathbf{p}_{X}$ of the debris. In this case, the rescattering wave function can be approximated by its eikonal form [in terms of $T$ matrix: $T\left(\mathbf{p}_{X}, \mathbf{k}_{i} ; \mathbf{p}_{X}^{\prime}, \mathbf{k}_{i}^{\prime}\right) \rightarrow T^{e i k}\left(\mathbf{p}_{X}\right)$ ], which allows one to describe the propagation of the debris produced after the $\gamma^{*}$ absorption by a target quark, while both hadronization processes and interactions between the newly produced prehadrons and the spectator nucleons take place. This series of soft interactions with the spectator system can be characterized by an effective cross section $\sigma_{\text {eff }}\left(z, Q^{2}, x\right)$, which depends upon time (or the distance $z$ traveled by the system $X$ ). Such an effective cross section allows one to construct a realistic profile function, which determines the eikonal approximation (see below) [29,31,34,50]. As a result, in presence of FSI, the PWIA overlaps given in Eq. (11) should be replaced by the suitable ones that encode FSI effects. In the $2 \mathrm{bbu}$ channel, where the asymptotic three-momentum of the spectator system is $\mathbf{P}_{A-1}=\mathbf{P}_{D}$, one has, for the matrix elements of the one-body current, an expression that has the schematic form

$$
\begin{aligned}
& \left\langle\hat{S}(1,2,3)\left\{\mathbf{p}_{X} ; \mathbf{P}_{D} \Psi_{D}\right\}\left|j_{\mu}^{N}\right| \Psi_{\mathrm{He}}\right\rangle \\
& \quad=\int d \mathbf{p}_{N}\left\langle\mathbf{p}_{X}\left|j_{\mu}^{N}\right| \mathbf{p}_{N}\right\rangle\left\langle\mathbf{p}_{N} ; \mathbf{P}_{D} \Psi_{D}\left|\hat{S}_{\mathrm{Gl}}(1,2,3)\right| \Psi_{\mathrm{He}}\right\rangle,
\end{aligned}
$$

where $\hat{S}_{\mathrm{Gl}}(1,2,3)$, represents the debris-nucleon eikonal scattering $S$ matrix, which depends upon the relative coordinates only, and it has been assumed to commute with $j^{\mu}$. This leads to consider overlaps like $\left\langle\mathbf{p}_{N} ; \mathbf{P}_{D} \Psi_{D}\left|\hat{S}_{\mathrm{Gl}}(1,2,3)\right| \Psi_{\mathrm{He}}\right\rangle$ (cf. Eq. (6) in Ref. [29]). The operator $\hat{S}_{\mathrm{Gl}}(1,2,3)$ can be written as

$$
\hat{S}_{\mathrm{Gl}}\left(\mathbf{r}_{1}, \mathbf{r}_{2}, \mathbf{r}_{3}\right)=\prod_{i=2,3}\left[1-\theta\left(z_{i}-z_{1}\right) \Gamma\left(\mathbf{b}_{1}-\mathbf{b}_{i}, z_{1}-z_{i}\right)\right],
$$

where $\mathbf{b}_{i}$ and $z_{i}$ are the perpendicular and parallel components of $\mathbf{r}_{i}$ (remember that $\mathbf{r}_{1}+\mathbf{r}_{2}+\mathbf{r}_{3}=0$ ) with respect to the direction of the propagation of the debris $\mathbf{p}_{X}$. In the DIS limit $\mathbf{p}_{X} \simeq \mathbf{q}$ and the eikonal $S$ matrix is defined with respect to $\mathbf{q}$. 
This implies that a dependence upon $\mathbf{q}$ has to be taken into account, but it is not explicitly indicated to avoid a too heavy notation; however, this will be recalled at the proper places. The profile function, $\Gamma$, is given by

$$
\Gamma\left(\mathbf{b}_{1 i}, z_{1 i}\right)=\frac{(1-i \alpha) \sigma_{\text {eff }}\left(z_{1 i}\right)}{4 \pi b_{0}^{2}} \exp \left[-\frac{\mathbf{b}_{1 i}^{2}}{2 b_{0}^{2}}\right],
$$

where $\mathbf{r}_{1 i}=\left\{\mathbf{b}_{1 i}, \mathbf{z}_{1 i}\right\}$, with $\mathbf{z}_{1 i}=\mathbf{z}_{1}-\mathbf{z}_{i}$ and $\mathbf{b}_{1 i}=\mathbf{b}_{1}-\mathbf{b}_{i}$. It can be seen that, in the present GEA, unlike in the standard Glauber approach, the profile function $\Gamma$ depends not only upon the transverse relative separation but also upon the longitudinal separation $z_{1, i}$ owing to the $z$ (or time) dependence of the effective cross section $\sigma_{\mathrm{eff}}\left(z_{1 i}\right)$ and the causal $\theta$ function, $\theta\left(z_{i}-z_{1}\right)$. In principle, the effective cross section, $\sigma_{\text {eff }}\left(z_{1 i}\right)$ also depends on the total energy of the debris, $W^{2} \equiv P_{X}^{2}=$ $\left(p_{N}+q\right)^{2}$. However, if the energy is not too large and the hadronization process takes place inside the nucleus $(A-1)$, the dependence on $W^{2}$ is weak, and the number of produced hadrons can be taken to be constant.

Therefore, one can assume $\sigma_{\text {eff }}\left(z_{1 i}, x_{\mathrm{Bj}}, Q^{2}\right) \sim$ $\sigma_{\text {eff }}\left(z_{1 i}\right) \quad[29,50]$. Let us briefly discuss the parameters entering Eq. (42), i.e., the profile function adopted in our model. Indeed, it is the same expression exploited in Ref. [29] to nicely describe the data measured at JLab [50], corresponding to the unpolarized spectator SiDIS off the deuteron. Also, the numerical values of the parameters present in Eq. (42) are the same as in Ref. [29], and obviously, given the lack of data for the polarized case (as well as theoretical calculations), the choice should be considered as a conservative one. In particular, $\sigma_{\text {eff }}$ is the cross section for the interaction of the formed hadrons (assumed to be a nucleon and a few pions) with the remnant nucleons. Hence, $\sigma_{\text {eff }}$ is given by the sum of two contributions: (i) the nucleon-nucleon cross section, $\sigma_{N N}$, and (ii) $\sigma_{\pi N} N_{\pi}^{\text {eff }}$, i.e., the nucleon-pion cross section times the effective number of pions which are produced, $N_{\pi}^{\text {eff }}$ (cf. Ref. [31] for the explicit evaluation). As to the parameter $\alpha$ in Eq. (42), it represents the ratio of the real to the imaginary part of the forward-scattering amplitude of the process. At the energies we are considering, $\alpha$ is basically the same for $N N$ and for $\pi N$ interactions, and in our calculation it has been taken constant, namely, $\alpha=-0.5$, following Ref. [29]. Finally, the slope parameter, $b_{0}$, is determined by the optical theorem, which yields $b_{0}^{2}=\sigma_{\text {eff }}^{2}\left(1+\alpha^{2}\right) /\left[16 \pi \sigma^{\mathrm{el}}\right]$, where $\sigma^{\text {el }}$ is the elastic part of the total effective cross section $\sigma_{\text {eff. }}$ The values we used for the relevant cross sections are the same as in Ref. [29], i.e., $\sigma_{N N}^{\mathrm{el}}=20 \mathrm{mb}, \sigma_{\pi N}^{\mathrm{el}}=10 \mathrm{mb}$, $\sigma_{N N}^{\text {tot }}=40 \mathrm{mb}, \sigma_{\pi N}^{\text {tot }}=25 \mathrm{mb}$. As a general remark, it should be pointed out that GEA has been successfully applied for studying at high energy both s.i. and exclusive quasielastic scattering off nuclei (see, e.g., Refs. [51-53]).

In conclusion, within the adopted approximation, the overlaps that include the FSI effects are given by

$$
\begin{aligned}
\mathcal{O}_{\lambda \lambda^{\prime}}^{S_{A}(\mathrm{FSI})}\left(\mathbf{P}_{\mathbf{D}}, E_{2 \mathrm{bbu}}\right)= & \left\langle\hat{S}_{\mathrm{Gl}}(1,2,3)\left\{\Psi_{\mathbf{P}_{D}}, \lambda, \mathbf{p}_{N}\right\} \mid S_{A}, \Phi_{A}\right\rangle\left\langle\Phi_{A}, S_{A} \mid \hat{S}_{\mathrm{Gl}}(1,2,3)\left\{\Psi_{\mathbf{P}_{D}}, \lambda^{\prime}, \mathbf{p}_{N}\right\}\right\rangle \\
= & \sum_{M_{D}} \sum_{\{\alpha, \tilde{\alpha}\}}\left\langle X M_{X} L_{\rho} M_{\rho} \mid \frac{1}{2} M_{A}\right\rangle\left\langle\tilde{X} \tilde{M}_{X} \tilde{L}_{\rho} \tilde{M}_{\rho} \mid \frac{1}{2} M_{A}\right\rangle\left\langle j_{23} m_{23} \frac{1}{2} \lambda \mid X M_{X}\right\rangle\left\langle\tilde{j}_{23} \tilde{m}_{23} \frac{1}{2} \lambda^{\prime} \mid \tilde{X} \tilde{M}_{X}\right\rangle \\
& \times\left\langle l_{23} \mu_{23} 1 M_{S} \mid j_{23} m_{23}\right\rangle\left\langle\tilde{l}_{23} \tilde{\mu}_{23} \tilde{1} M_{S} \mid \tilde{j}_{23} \tilde{m}_{23}\right\rangle\left\langle L_{D} m_{L} 1 M_{S} \mid 1 M_{D}\right\rangle\left\langle\tilde{L}_{D} \tilde{m}_{L} \tilde{1} M_{S} \mid 1 M_{D}\right\rangle O_{\alpha}^{(\mathrm{FSI})}\left(\mathbf{P}_{D}, E_{2 \mathrm{bbu}}\right) \\
& \times O_{\tilde{\alpha}}^{(\mathrm{FSI})}\left(\mathbf{P}_{D}, E_{2 \mathrm{bbu}}\right),
\end{aligned}
$$

where

$$
O_{\alpha}^{(\mathrm{FSI})}\left(\mathbf{P}_{D}, E_{2 \mathrm{bbu}}\right)=\int d \boldsymbol{\rho} \int d \mathbf{r}_{23} e^{i \mathbf{P}_{D} \boldsymbol{\rho}} S_{\mathrm{Gl}}\left(\mathbf{r}_{23}, \boldsymbol{\rho}\right) \frac{\Psi_{L_{D}}\left(\left|\mathbf{r}_{23}\right|\right)}{\left|\mathbf{r}_{23}\right|} Y_{L_{D} m_{L}}\left(\hat{\mathbf{r}}_{23}\right) Y_{L_{\rho} M_{\rho}}(\hat{\boldsymbol{\rho}}) Y_{l_{23} \mu_{23}}\left(\hat{\mathbf{r}}_{23}\right) R_{\alpha}\left(\left|\mathbf{r}_{23}\right|,|\boldsymbol{\rho}|\right)
$$

with $S_{\mathrm{Gl}}\left(\mathbf{r}_{23}, \boldsymbol{\rho}\right)$ the nonsingular part of the matrix elements of $\hat{S}_{\mathrm{Gl}}(1,2,3)$ (remember that the adopted eikonal $S$ matrix is diagonal in the Jacobi-coordinate basis). A further issue is represented by the fact that the direction of the target polarization axis, $\hat{\mathbf{k}}_{e}$, is not totally parallel to the the direction which determines the eikonal $S$ matrix, i.e., $\hat{\mathbf{p}}_{X}$. Indeed, in the Bjorken limit, the momentum transfer $\mathbf{q}$ is almost parallel to the beam direction $\mathbf{k}_{e}$ so that in this case one can choose the quantization $z$ axis along the beam direction and perform calculations of FSI effects within such a coordinate system, because $\hat{\mathbf{k}}_{e} \simeq \hat{\mathbf{q}} \simeq \hat{\mathbf{p}}_{X}$. However, at finite values of $|\mathbf{q}|$, the beam direction differs from the direction which determines the eikonal $S$ matrix. To reconcile the polarization axis and the eikonal approximation, one needs to rotate the target wave function from the quantization axis of the polarization $\mathbf{S}_{A}$ to the system with the $z$ axis along $\mathbf{q}$, namely,

$$
\begin{aligned}
\left\langle\theta, \phi \mid \Psi_{{ }^{3} \mathrm{He}}\right\rangle_{\hat{\mathbf{S}}_{A}}= & \left\langle\theta^{\prime}, \phi^{\prime}|D(0, \alpha, 0)| \Psi_{{ }^{3} \mathrm{He}}\right\rangle_{\hat{\mathbf{q}}} \\
= & \cos (\alpha / 2)\left\langle\theta^{\prime}, \phi^{\prime} \mid \Psi_{{ }^{3} \mathrm{He}}^{\mathcal{M}=1 / 2}\right\rangle_{\hat{\mathbf{q}}} \\
& +\sin (\alpha / 2)\left\langle\theta^{\prime}, \phi^{\prime} \mid \Psi_{{ }^{3} \mathrm{He}}^{\mathcal{M}=-1 / 2}\right\rangle_{\hat{\mathbf{q}}},
\end{aligned}
$$

where the subscripts indicate the direction of the $z$ axis with $\cos \alpha=\hat{\mathbf{S}}_{A} \cdot \hat{\mathbf{q}}$. In this case, the tensor $W_{\mu \nu}^{\text {s.i. }}\left(S_{A}, Q^{2}, P_{h}\right)$ in Eq. (9) is modified and reads

$$
\begin{aligned}
W_{\mu \nu}^{\text {s.i. }}\left(S_{A}, Q^{2}, P_{h}\right)= & \cos ^{2}(\alpha / 2) W_{\mu \nu}^{\frac{1}{2} \frac{1}{2}}+\sin ^{2}(\alpha / 2) W_{\mu \nu}^{-\frac{1}{2}-\frac{1}{2}} \\
& +\sin \alpha\left[\frac{1}{2}\left(W_{\mu \nu}^{\frac{1}{2}-\frac{1}{2}}+W_{\mu \nu}^{-\frac{1}{2} \frac{1}{2}}\right)\right]
\end{aligned}
$$


where $W_{\mu \nu}^{\mathcal{M} M^{\prime}}$ are defined with respect to the new axis, i.e., parallel to q. Then, introducing the overlaps with quantization axis $\hat{\mathbf{q}}$,

$$
\begin{aligned}
\mathcal{O}_{\lambda \lambda^{\prime}}^{\mathcal{M} M^{\prime}(\mathrm{FSI})}\left(\mathbf{P}_{\mathbf{D}}, E_{2 \mathrm{bbu}}\right)= & \left\langle\hat{S}_{\mathrm{Gl}}(1,2,3)\left\{\Psi_{\mathbf{P}_{D}}, \lambda, \mathbf{p}_{N}\right\} \mid \Psi_{A}^{\mathcal{M}}\right\rangle_{\hat{\mathbf{q}}} \\
& \times\left\langle\Psi_{A}^{\mathcal{M}^{\prime}} \mid \hat{S}_{\mathrm{Gl}}(1,2,3)\left\{\Psi_{\mathbf{P}_{D}}, \lambda^{\prime}, \mathbf{p}_{N}\right\}\right\rangle_{\hat{\mathbf{q}}}
\end{aligned}
$$

and making use of their property under complex conjugation, namely,

$$
\mathcal{O}_{\lambda \lambda^{\prime}}^{\mathcal{M} M^{\prime}}\left(\mathbf{P}_{A-1}, E\right)=(-1)^{\mathcal{M}+\mathcal{M}^{\prime}+\lambda+\lambda^{\prime}}\left[\mathcal{O}_{-\lambda-\lambda^{\prime}}^{-\mathcal{M}-\mathcal{M}^{\prime}}\left(\mathbf{P}_{A-1}, E\right)\right]^{*},
$$

it can be shown that the contribution to the distorted spindependent spectral function owing to the target polarization takes the form

$$
\mathcal{P}_{(\mathrm{FSI})}^{\hat{\mathrm{S}}_{A}}=\cos \alpha \mathcal{P}_{(\mathrm{FSI})}^{\frac{1}{2} \frac{1}{2}}+\sin \alpha \mathcal{P}_{(\mathrm{FSI})}^{\frac{1}{2}-\frac{1}{2}},
$$

where $\mathcal{P}_{(\mathrm{FSI})}^{\mathcal{M} \mathcal{M}^{\prime}}$ are evaluated with quantization axis $\hat{\mathbf{q}}$ and the relations $\mathcal{P}_{(\mathrm{FSI})}^{-\frac{1}{2}-\frac{1}{2}}=-\mathcal{P}_{(\mathrm{FSI})}^{\frac{1}{2} \frac{1}{2}}$ and $\mathcal{P}_{(\mathrm{FSI})}^{-\frac{1}{2} \frac{1}{2}}=\mathcal{P}_{(\mathrm{FSI})}^{\frac{1}{2}-\frac{1}{2}}$ have been exploited (see Ref. [15]). As happens in PWIA, $\mathcal{P}_{(\mathrm{FSI})}^{\mathcal{M} M^{\prime}}$ can be can be decomposed as

$$
\mathcal{P}_{(\mathrm{FSI})}^{\mathcal{M} M^{\prime}}=\mathcal{P}_{\|(\mathrm{FSI})}^{\mathcal{M} M^{\prime}} \mathbf{e}_{0}+\mathcal{P}_{1 \perp(\mathrm{FSI})}^{\mathcal{M} M^{\prime}} \mathbf{e}_{+}+\mathcal{P}_{2 \perp(\mathrm{FSI})}^{\mathcal{M} M^{\prime}} \mathbf{e}_{-},
$$

where $\mathcal{P}_{\|(\perp)}^{\mathcal{M} M}$ are defined in full analogy with Eq. (34), while $\mathcal{P}_{\|(\perp)}^{\mathcal{M}-M}$ are given by

$$
\begin{aligned}
\mathcal{P}_{||(\mathrm{FSI})}^{\frac{1}{2}-\frac{1}{2}} & =\frac{1}{2}\left[\mathcal{O}_{\frac{1}{2} \frac{1}{2}}^{\frac{1}{2}-\frac{1}{2}(\mathrm{FSI})}-\mathcal{O}_{-\frac{1}{2}-\frac{1}{2}}^{\frac{1}{2}-\frac{1}{2}(\mathrm{FSI})}+\mathrm{c.c} .\right], \\
\mathcal{P}_{1 \perp(\mathrm{FSI})}^{\frac{1}{2}-\frac{1}{2}} & =-\frac{1}{\sqrt{2}}\left[\mathcal{O}_{\frac{1}{2}-\frac{1}{2}}^{\frac{1}{2}-\frac{1}{2}(\mathrm{FSI})}+\mathcal{O}_{-\frac{1}{2} \frac{1}{2}}^{* \frac{1}{2}-\frac{1}{2}(\mathrm{FSI})}\right], \\
\mathcal{P}_{2 \perp(\mathrm{FSI})}^{\frac{1}{2}-\frac{1}{2}} & =\frac{1}{\sqrt{2}}\left[\mathcal{O}_{-\frac{1}{2} \frac{1}{2}}^{\frac{1}{2}-\frac{1}{2}(\mathrm{FSI})}+\mathcal{O}_{\frac{1}{2}-\frac{1}{2}}^{* \frac{1}{2}-\frac{1}{2}(\mathrm{FSI})}\right] .
\end{aligned}
$$

In what follows, for the sake of brevity, the diagonal components are indicated by only one projection, i.e., $\mathcal{P}^{\mathcal{M M}}\left(\mathcal{O}^{\mathcal{M} M}\right) \rightarrow \mathcal{P}^{\mathcal{M}}\left(\mathcal{O}^{\mathcal{M}}\right)$.

\section{NUMERICAL RESULTS AND DISCUSSION}

In this section, numerical calculations of the distorted spindependent spectral function in the $2 \mathrm{bbu}$ channel are presented. Particular attention is paid to two typical kinematics, known as parallel $\left(\hat{\mathbf{p}}_{N} \| \hat{z}\right.$, with $\left.\hat{z} \equiv \hat{\mathbf{q}}\right)$ and perpendicular $\left(\hat{\mathbf{p}}_{N} \perp \hat{z}\right)$ kinematics. In the unpolarized case, the spectral function within these two kinematics is influenced by rather different physical effects. Namely, in the parallel kinematics, FSIs are found to be negligibly small and, accordingly, the process is suitable for studying the DIS structure function of a bound nucleon; however, in the perpendicular kinematics the FSI effects are predominant, so that details of the hadronization mechanism can be probed [29]. Bearing this in mind, let us consider the spectator SiDIS by a polarized target. Usually, all quantities are presented in terms of the asymptotic threemomentum of the spectator system, $\mathbf{P}_{A-1}$. Moreover, to keep the notation as close as possible to the one in the quasielastic

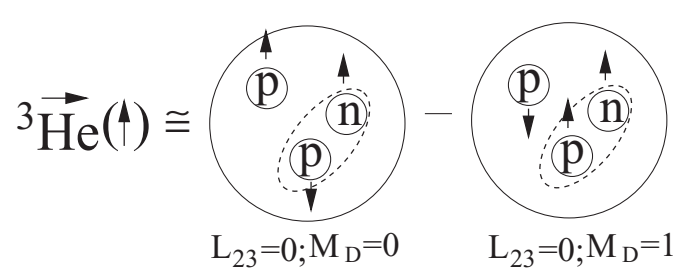

FIG. 4. In a polarized ${ }^{3} \mathrm{He}$ the total spin of the proton pair is basically zero, so that the neutron spin is mainly directed along the target polarization. The proton-neutron spectator pair " 23 " forms the deuteron with $L_{23} \sim 0$ in the final state. For an easy representation, the contribution of the $D$ state is not depicted.

$A\left(e, e^{\prime} p\right)$ reactions, we introduce the missing momentum $\mathbf{p}_{\text {mis }} \equiv \mathbf{P}_{A-1}$. Before going into the numerical analysis, let us have a qualitative glance at the intrinsic structure of a polarized ${ }^{3} \mathrm{He}$ nucleus (that represents our test ground). It is known that such a nucleus basically represents a "polarized neutron." As a matter of fact, in the polarized ${ }^{3} \mathrm{He}$ the spin projections of the protons almost $(\sim 90 \%)$ cancel each other, and the nuclear polarization is governed by that of the neutron [11] (see Fig. 4). This implies that, within PWIA when a deuteron acts as a spectator, the spin of the neutron in the final deuteron is expected to be directed along its initial polarization, i.e., along the polarization of the target. Correspondingly, the parallel component of the spin-dependent spectral function, $\mathcal{P}_{\|}^{\frac{1}{2}}=\mathcal{O}_{\frac{1}{2} \frac{1}{2}}^{\frac{1}{2}}-\mathcal{O}_{-\frac{1}{2}-\frac{1}{2}}^{\frac{1}{2}}$, gets the main contribution from the deuteron configurations with $M_{D}=0$ and $M_{D}=1$. This can be easily understood considering only the deuteron $S$ wave in Eq. (43). Indeed, putting $L_{\rho}=L_{D}=l_{23}=0$, one can see that in $\mathcal{O}_{\frac{1}{2} \frac{1}{2}}^{\frac{1}{2}}$ the component with $M_{D}=0$ contributes and in $\mathcal{O}_{-\frac{1}{2}-\frac{1}{2}}^{\frac{1}{2}}$ the component with $M_{D}=1$ acts [cf. Eq. (39)]. Moreover, it turns out that the contributions from $M_{D}=0$ (with an upward neutron polarization) and from $M_{D}=1$ have relative size $1 / 2: 1$, so that $\mathcal{P}_{\|}^{\frac{1}{2}} \simeq-\frac{1}{2} \mathcal{O}_{-\frac{1}{2}-\frac{1}{2}}^{\frac{1}{2}}$ and is negative. Although the presence of (i) $P$ and $D$ waves in ${ }^{3} \mathrm{He}$ and (ii) the $D$ wave in the deuteron changes the simple scenario depicted in Fig. 4, at low missing momenta one still expects that $\mathcal{P}_{\|}^{\frac{1}{2}} \simeq-\frac{1}{2} \mathcal{O}_{-\frac{1}{2}-\frac{1}{2}}^{\frac{1}{2}}$.

In Fig. 5, the absolute value of $\mathcal{P}^{\frac{1}{2}}$ is shown as a function of the missing momentum in both the parallel $\left(\theta_{\text {mis }}=180^{\circ}, \phi_{\text {mis }}=180^{\circ}\right)$ and the perpendicular $\left(\theta_{\text {mis }}=90^{\circ}\right.$ and $\phi_{\text {mis }}=180^{\circ}$ ) kinematics. The dashed lines correspond to the PWIA case; the solid line is $\left|\mathcal{P}_{\|}^{\frac{1}{2}}\right|$ with FSI effects included. As mentioned above, $\mathcal{P}_{\|}^{\frac{1}{2}}$ at low missing momenta is negative within both kinematics, as indicated by the inset "minus" sign. In the parallel kinematics (left panel) at moderate values of $\left|\mathbf{p}_{\text {mis }}\right| \sim 2 \mathrm{fm}^{-1}, \mathcal{P}_{\|}^{\frac{1}{2}}$ in PWIA vanishes and at higher $\left|\mathbf{p}_{\text {mis }}\right|>2 \mathrm{fm}^{-1}$ becomes positive. This is an important feature of the parallel component of the spin-dependent spectral function because, as seen from Fig. 5, $\mathcal{P}_{\|}^{\frac{1}{2}}$ with FSI effects never changes the sign, in both kinematics. This can be exploited to determine the presence (and strength) of FSI. Notice that, similarly to the unpolarized case [29], FSIs 


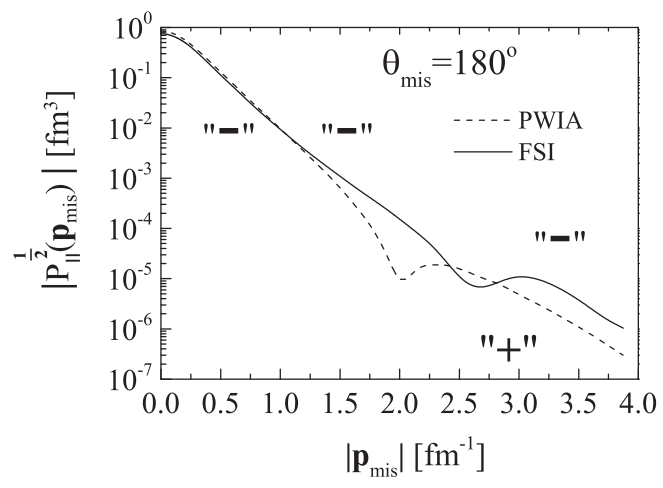

(a)

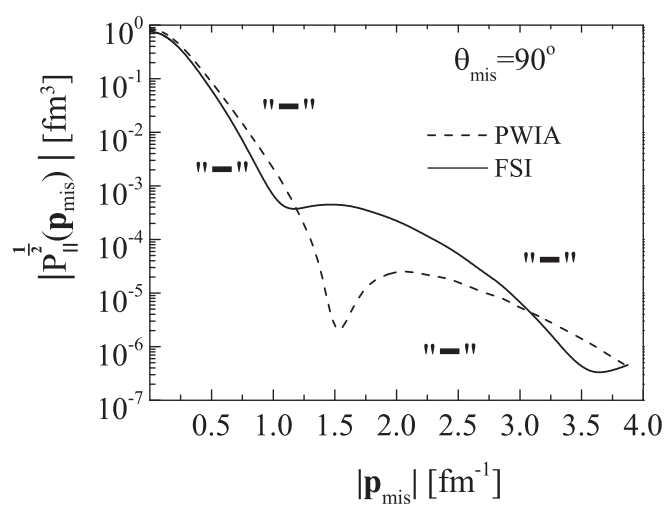

(b)

FIG. 5. The absolute value $\left|\mathcal{P}_{\|}^{\frac{1}{2}}\right|$, relevant for a spectator SiDIS with a deuteron in the final state, for the reaction ${ }^{3} \mathrm{He}\left(\vec{e}, e^{\prime 2} H\right) X$, in the Bjorken limit, vs the missing momentum $\left(\mathbf{p}_{\text {mis }} \equiv \mathbf{P}_{D}\right.$ ), in parallel, $\theta_{\text {mis }}=180^{\circ}$ and $\phi_{\text {mis }}=180^{\circ}$ (left), and perpendicular, $\theta_{\text {mis }}=90^{\circ}$ and $\phi_{\text {mis }}=180^{\circ}$ (right) kinematics. Dashed line, PWIA calculations; solid line, calculations with FSI effects. The inset symbols, + and - , indicate the sign of $\mathcal{P}_{\|}^{\frac{1}{2}}$. Notice that $\mathcal{P}_{\|}^{\frac{1}{2}}$ with FSI effects remains always negative, while $\mathcal{P}_{\|}^{\frac{1}{2}}$ in PWIA changes sign only in parallel kinematics.

are negligible at low values of $\left|\mathbf{p}_{\text {mis }}\right|$ (because in this case one has a fast final debris, given $\mathbf{p}_{X} \sim \mathbf{q}$ ), while the FSI contribution becomes sizable for $\left|\mathbf{p}_{\text {mis }}\right| \geqslant 1 \mathrm{fm}^{-1}$, where the equal sign holds for the perpendicular kinematics (right panel). Furthermore, because of the nontrivial angular dependence in $\mathcal{P}_{\|}^{\frac{1}{2}}$ (cf. Fig. 6), though in PWIA there is a zero at $\left|\mathbf{p}_{\text {mis }}\right| \sim 2 \mathrm{fm}^{-1}$ in parallel kinematics and a minimum at $\left|\mathbf{p}_{\text {mis }}\right| \sim 1.5 \mathrm{fm}^{-1}$ in perpendicular kinematics, the magnitude of FSI effects is much larger in this last setting (cf. Fig. 4 of Ref. [29]).

In Fig. 6 we present the angular dependence of $\mathcal{P}_{\|}^{\frac{1}{2}}\left(\mathbf{p}_{\text {mis }}\right)$ for fixed values of the missing momentum: $\left|\mathbf{p}_{\text {mis }}\right|=1 \mathrm{fm}^{-1}$ (left panel) and $\left|\mathbf{p}_{\text {mis }}\right|=1.8 \mathrm{fm}^{-1}$ (right panel). The choice $\left|\mathbf{p}_{\text {mis }}\right|=$ $1 \mathrm{fm}^{-1}$ has been inspired by the fact that, as seen from Fig. 5, FSI effects are still negligibly small (at least in the parallel kinematics), whereas $\left|\mathbf{p}_{\text {mis }}\right|=1.8 \mathrm{fm}^{-1}$ corresponds to the region where the PWIA spectral function has a minimum; hence, the FSI effects are maximized. It can be seen that, at lower missing momenta, FSI effects are small and in the backward hemisphere they can be safely neglected; at $\left|\mathbf{p}_{\text {mis }}\right|=1.8 \mathrm{fm}^{-1}$ the effects of FSI are considerable, even predominant, in the whole range of the missing angle. It is worth noting that Figs. 5 and 6 can offer hints for choosing the kinematics for both spectator and standard SiDIS, to minimize or maximize FSI effects. Let us remember that for the spectator SiDIS in the first case one can address the structure functions of bound nucleons and in the second kinematics the hadronization mechanism can be probed (see below).

Let us now briefly discuss the perpendicular components of the spin-dependent spectral function; see Eqs. (33), (34), and (51). In the Bjorken limit, when $\hat{\mathbf{q}}$ becomes parallel to the $z$ axis, that in our analysis is also the target polarization axis, i.e., $\hat{z} \| \mathbf{S}_{A}$, one can clearly see from Eq. (35) that $\mathcal{P}^{\hat{\mathbf{S}}_{A}}$ in PWIA is exactly zero within both the parallel (i.e., $\hat{\mathbf{p}}_{N} \| \mathbf{S}_{A}$ ) and the perpendicular (i.e., $\hat{\mathbf{p}}_{N} \perp \mathbf{S}_{A}$ ) kinematics [recall that only the term $\hat{\mathbf{p}}_{N}\left(\hat{\mathbf{p}}_{N} \cdot \mathbf{S}_{A}\right) B_{2}\left(\left|\mathbf{p}_{N}\right|, E\right)$ can contribute to the perpendicular component of the spectral function, because the term proportional to $\mathbf{S}_{A} B_{1}\left(\left|\mathbf{p}_{N}\right|, E\right)$ can contribute only

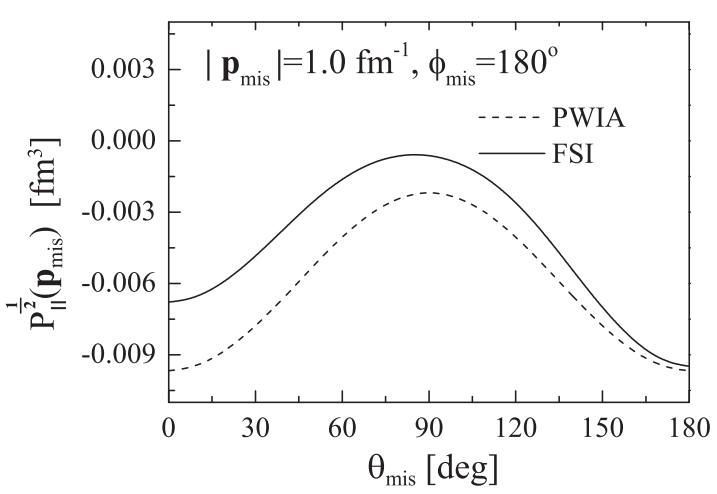

(a)

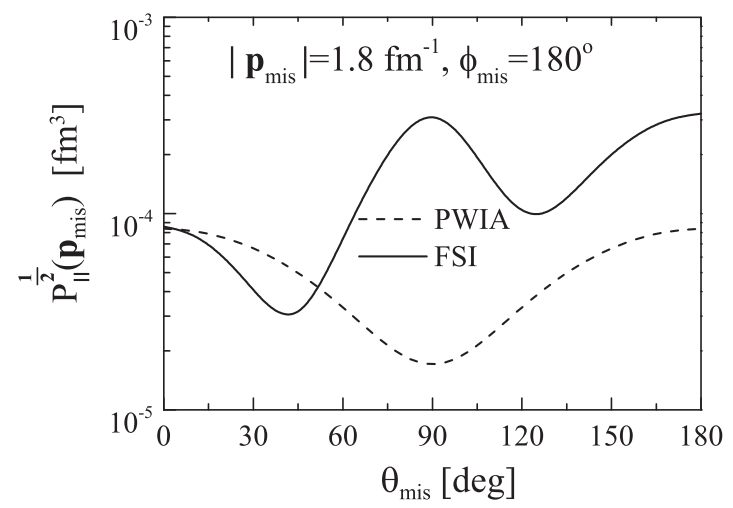

(b)

FIG. 6. The angular dependence of $\mathcal{P}_{\|}^{\frac{1}{2}}$ for two values of the missing momentum. Dashed lines correspond to the PWIA calculations. Solid lines include FSI effects. 


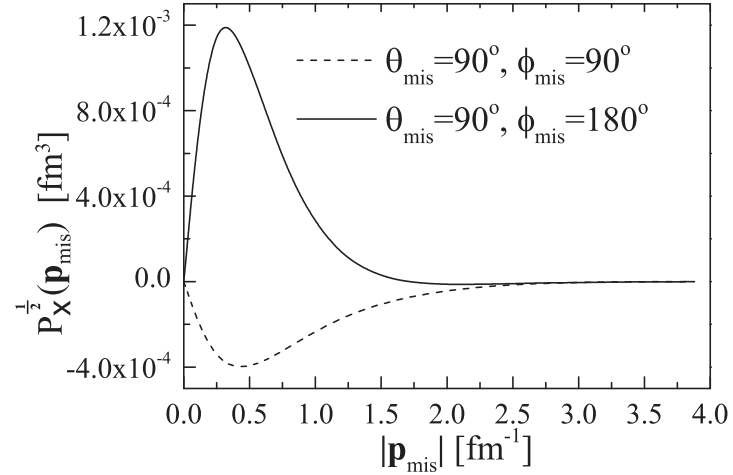

(a)

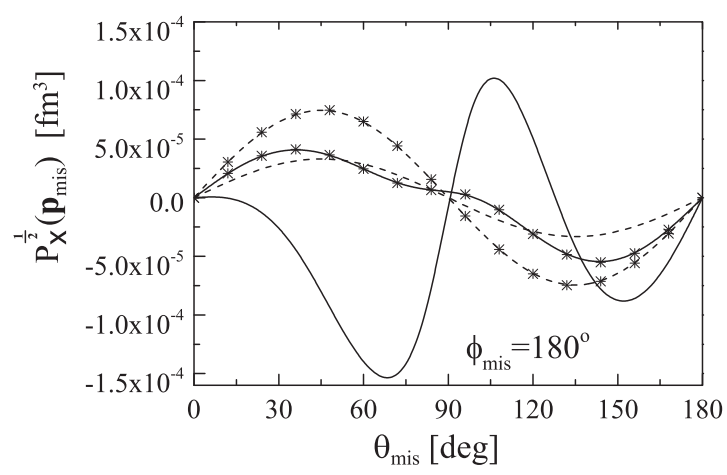

(b)

FIG. 7. The $x$ component of the spin-dependent spectral function, relevant for a spectator SiDIS with a deuteron in the final state. (Left) $\mathcal{P}_{x}^{\frac{1}{2}}\left(\mathbf{p}_{\text {mis }}\right)$ vs $|\mathbf{p}|_{\text {mis }}$, in perpendicular kinematics, $\theta_{\text {mis }}=90^{\circ}$, for two values of $\phi_{\text {mis }}, 180^{\circ}$ (solid line) and $90^{\circ}$ (dashed line). Within such a kinematics the PWIA spectral function is exactly zero, so that the $\mathcal{P}_{x}^{\frac{1}{2}}\left(\mathbf{p}_{\text {mis }}\right)$ is entirely attributable to the FSI effects. (Right) Angular dependence of $\mathcal{P}_{x}^{\frac{1}{2}}\left(\mathbf{p}_{\text {mis }}\right)$ for two values of the missing momentum, $\left|\mathbf{p}_{\text {mis }}\right|=0.5 \mathrm{fm}^{-1}$ (crossed lines) and $\left|\mathbf{p}_{\text {mis }}\right|=1.8 \mathrm{fm}{ }^{-1}$. Dashed lines, PWIA; solid lines, FSI effects are taken into account. Notice that, for a convenient presentation, the results corresponding to $\left|\mathbf{p}_{\text {mis }}\right|=0.5 \mathrm{fm}^{-1}$ have been rescaled by a factor $2 \times 10^{-2}$.

to the parallel one]. ${ }^{1}$ However, in presence of FSI, the spectral function depends also upon the vector $\mathbf{q}$ [cf. below Eq. (41)], so that in Eq. (35) terms proportional to q must be included. In particular, a term like $\sim \hat{\mathbf{p}}_{N}\left(\mathbf{q} \cdot \mathbf{S}_{A}\right)$ will contribute in the perpendicular kinematics, causing $\mathcal{P}_{\perp}^{\hat{\mathbf{S}}_{A}}$ to be different from zero. Therefore, a nonzero value of $\mathcal{P}_{1(2) \perp}^{\hat{\mathbf{S}}_{A}}$, in the perpendicular kinematics, undoubtedly points to FSI effects. Such a qualitative result can be obtained in a more rigorous way by closely inspecting Eq. (36) and investigating the dependence upon both $\phi_{\text {mis }}$ and $\theta_{\text {mis. }}$. It can be seen that the dependence upon $\phi_{\text {mis }}$ is determined by $M_{\rho}-\tilde{M}_{\rho}=$ $\lambda^{\prime}-\lambda$. This means that the parallel spectral function does not depend at all upon $\phi_{\text {mis }}$, while the $\phi_{\text {mis }}$ dependence of the perpendicular spectral function will be $\mathcal{P}_{1(2) \perp}^{\hat{\mathrm{S}}_{A}} \sim \exp \left( \pm i \phi_{\text {mis }}\right)$. Moreover, the presence of the term $\sim Y_{L_{\rho} M_{\rho}}\left(\hat{\mathbf{p}}_{N}\right) \mathrm{Y}^{*} \tilde{L}_{\rho} \tilde{M}_{\rho}\left(\hat{\mathbf{p}}_{N}\right)$ demonstrates that $\mathcal{P}_{1(2) \perp}^{\hat{\boldsymbol{S}}_{A}}$ identically vanish at $\theta_{\text {mis }}=0, \pi / 2, \pi$ in PWIA, because $M_{\rho}-\tilde{M}_{\rho}= \pm 1$. As is well known, the two spherical harmonics can be expanded on terms like $\left\langle L_{\rho} M_{\rho} \tilde{L}_{\rho}-\tilde{M}_{\rho} \mid \mathcal{L} \pm 1\right\rangle Y_{\mathcal{L} \pm 1}\left(\hat{\mathbf{p}}_{N}\right)$ that vanish for $\theta_{\text {mis }}=0, \pi$. For $\theta_{\text {mis }}=\pi / 2$ the argument is less direct. The two spherical harmonics are different from zero only if $\tilde{L}_{\rho}+\tilde{M}_{\rho}$ and $L_{\rho}+M_{\rho}$ are both even, but $\tilde{L}_{\rho}+L_{\rho}$ is even and $\tilde{M}_{\rho}+M_{\rho}$ is odd. When the FSI effects are taken in to account, the previous product is replaced with $\int d \rho_{\perp} \ldots J_{\mathcal{M}}\left(\left|\mathbf{p}_{\text {mis } \perp}\right| \rho_{\perp}\right)$. $\int d \tilde{\rho}_{\perp} \cdots J_{\mathcal{M}^{\prime}}\left(\left|\mathbf{p}_{\text {mis } \perp}\right| \tilde{\rho}_{\perp}\right)$, where $J_{\mathcal{M}\left(\mathcal{M}^{\prime}\right)}$ are the cylindrical Bessel functions and one has still $\mathcal{M}-\mathcal{M}^{\prime}= \pm 1$. It is clear that, in the parallel kinematics (i.e., $\mathbf{p}_{\text {mis } \perp}=0$ ), at most only one Bessel function cannot vanish $\left[J_{\mathcal{M}}(0) \neq 0\right.$ only for

\footnotetext{
${ }^{1}$ One should not confuse the perpendicular and parallel kinematics, which refer to the direction of nucleon momentum $\mathbf{p}_{N}$, with the parallel and perpendicular components of the spectral function, which refer to the direction of the vector $\mathcal{P}^{\hat{\mathbf{s}}_{A}}$.
}

$\mathcal{M}=0$ ] and therefore $\mathcal{P}_{\perp}^{\hat{\mathbf{S}}_{A}}$ is zero even in presence of FSI for $\mathbf{p}_{\text {mis } \perp}=0$.

One should notice that for the spherical components one has $\mathcal{P}_{1 \perp}^{\hat{\mathbf{S}}_{A}}=\left[\mathcal{P}_{2 \perp}^{\hat{\mathbf{S}}_{A}}\right]^{*}$ [see Eq. (33)], but for numerical analysis it is more convenient to deal with real quantities, e.g., with the Cartesian components $\mathcal{P}_{x}^{\hat{\mathbf{S}}_{A}}$ and $\mathcal{P}_{y}^{\hat{\mathbf{S}}_{A}}$; see Eq. (34). Because $\mathcal{P}_{y}^{\hat{\mathbf{S}}_{A}}\left(\phi_{\text {mis }}\right)=-\mathcal{P}_{x}^{\hat{\mathbf{S}}_{A}}\left(\pi / 2+\phi_{\text {mis }}\right)$, it is sufficient to analyze only one component, say $\mathcal{P}_{x}^{\hat{\mathbf{S}}_{A}}$. In the left panel of Fig. 7, the Bjorken limit of $\mathcal{P}_{x}^{\frac{1}{2}}\left(\phi_{\text {mis }}\right)$ is shown as a function of $\left|\mathbf{p}_{\text {mis }}\right|$, in the perpendicular kinematics and two values of $\phi_{\text {mis. }}$. It can be seen that $\mathcal{P}_{x}^{\frac{1}{2}}$, in comparison with $\mathcal{P}_{\|}^{\frac{1}{2}}$ (Fig. 6), is negligibly small, and in the perpendicular kinematics is entirely governed by FSI. In the right panel of Fig. 7, the angular dependence of $\mathcal{P}_{x}^{\frac{1}{2}}$, both without and with FSI effects, is presented for two values of missing momentum. As already mentioned, the PWIA calculations vanish at $\theta_{\text {mis }}=0, \pi / 2, \pi$. Moreover, it is seen that the $x$ component is much smaller then the parallel $\mathcal{P}_{\|}^{\frac{1}{2}}$ in the whole range of $\theta_{\text {mis }}$. Finally, from Fig. 7, one could get the impression that, at $\theta_{\text {mis }}=90^{\circ}$ the $x$ component of the spin-dependent spectral functions vanishes both without and with FSI effects. This is because of the adopted linear scale of the figure. Actually, while in PWIA $\mathcal{P}_{x}^{\frac{1}{2}}$ is exactly zero, the calculations with FSI show that $\mathcal{P}_{x}^{\frac{1}{2}}\left(\theta_{\text {mis }}=90^{\circ}\right) \sim 10^{-3}$.

\section{A. Cross sections and asymmetries}

In this section, the relevance of different components of the spin-dependent spectral function is analyzed with respect to the application in spectator SiDIS processes with polarized particles. For the sake of brevity, in what follows the notation $\mathcal{K} \equiv\left(k_{e}+k_{e}^{\prime}\right)$ is used. In the DIS limit, because of the scaling phenomenon, instead of the structure functions $G_{1,2}$ one introduces the more familiar 
scaling functions $g_{1 N}\left(x_{N}\right)=\left(p_{N} q\right) G_{1}^{N}\left(Q^{2}, p_{N} q\right)$ and $g_{2 N}\left(x_{N}\right)=\left(p_{N} q\right)^{2} / m_{N}^{2} G_{2}\left(Q^{2}, p_{N} q\right)$, with $x_{N}=Q^{2} / 2 q p_{N}$ It can be shown that in the leading-twist approximation the contribution of the structure function $g_{2 N}\left(x_{N}\right)$ to the cross section vanishes and only $g_{1 N}$ is relevant to describe the antisymmetric part of the tensor $w_{\mu \nu}^{\text {DIS }}$ [Eq. (18)]. Therefore, the contraction of the antisymmetric nuclear tensor with the leptonic one results in

$$
\begin{aligned}
L^{\mu \nu}\left(h_{e}\right) W_{\mu \nu}^{a, \text { s.i. }}\left(S_{A}, Q^{2}, P_{D}\right) & \sim-2 h_{e} \frac{m_{N}}{2 E_{N}} Q^{2} G_{1}^{N}\left(Q^{2}, p_{N} q\right) \mathcal{K}^{\beta}\left(\mathcal{B}_{\beta} \cdot \mathcal{P}^{\hat{\mathbf{s}}_{A}}\right) \\
& \equiv h_{e} \frac{Q^{2} m_{N}}{E_{N} q p_{N}} g_{1 N}\left(x_{N}\right)\left[\left(\mathcal{K} \cdot \mathcal{P}^{\hat{\mathbf{S}}_{A}}\right)+\frac{\left(\mathcal{K} \cdot \mathbf{p}_{N}\right)}{m_{N}} \frac{\left(\mathbf{p}_{N} \cdot \mathcal{P}^{\hat{\mathbf{S}}_{A}}\right)}{E_{N}+m_{N}}-\mathcal{K}_{0} \frac{\left(\mathbf{p}_{N} \cdot \mathcal{P}^{\hat{\mathbf{S}}_{A}}\right)}{m_{N}}\right] .
\end{aligned}
$$

To experimentally single out the spin-dependent part of the cross section, one measures asymmetries of the cross sections corresponding to the scattering of electrons with opposite helicities. In particular, the asymmetry

$$
\begin{aligned}
\frac{\Delta \sigma^{\hat{\mathbf{s}}_{A}}}{d \varphi_{e} d x_{\mathrm{Bj}} d y d \mathbf{P}_{D}} & \equiv \frac{d \sigma^{\hat{\mathbf{s}}_{A}}\left(h_{e}=1\right)-d \sigma^{\hat{\mathbf{s}}_{A}}\left(h_{e}=-1\right)}{d \varphi_{e} d x_{\mathrm{Bj}} d y d \mathbf{P}_{D}} \\
& =4 \frac{\alpha_{\mathrm{em}}^{2}}{Q^{2} z_{N} \mathcal{E}} \frac{m_{N}}{E_{N}} g_{1 N}\left(\frac{x_{\mathrm{Bj}}}{z_{N}}\right)\left[\left(\mathcal{K} \cdot \mathcal{P}^{\hat{\mathbf{s}}_{A}}\right)+\frac{\left(\mathcal{K} \cdot \mathbf{p}_{N}\right)}{m_{N}} \frac{\left(\mathbf{p}_{N} \cdot \mathcal{P}^{\hat{\boldsymbol{s}}_{A}}\right)}{E_{N}+m_{N}}-\mathcal{K}_{0} \frac{\left(\mathbf{p}_{N} \cdot \mathcal{P}^{\hat{\mathrm{s}}_{A}}\right)}{m_{N}}\right],
\end{aligned}
$$

allows one to single out the spin-dependent part of the cross section in the spectator SiDIS with a detected deuteron. In Eq. (53), we intentionally left the subscript $N$, because it can be applied to both $A=3$ polarized targets: ${ }^{3} \mathrm{He}$ and ${ }^{3} \mathrm{H}$. However, the actual calculations have been performed for ${ }^{3} \overrightarrow{\mathrm{He}}$ and therefore $N$ has to be substituted by $p$, i.e., $g_{1 N} \rightarrow g_{1 p}$. In Eq. (53) $z_{N}$ is proportional to the light-cone fraction of the momentum carried by the nucleon in the nucleus, i.e., $z_{N}=\left(p_{N} q\right) / m_{N} v \approx 1-p_{N 3} / m_{N} \approx A p_{N}^{+} / P_{A}^{+}$. It yields a measure of the difference between $x_{\mathrm{Bj}}$ and $x_{N}$, which is the actual variable upon which $g_{1 N}$ depends. The asymmetry in Eq. (53) has a factorized form, where the electromagnetic part of the interaction (the spin-dependent structure function $g_{1 N}$ ) is separated from the nuclear-structure effects (the term inside the square brackets, proportional to the spin-dependent spectral function $\mathcal{P}^{\hat{\mathrm{S}}_{A}}$ ) and some kinematical factors. Such a form shows that, in principle, one can experimentally achieve a direct access to the spin-dependent structure function of a bound nucleon, provided that the expression within the square brackets can be reliably calculated. Indeed, in such a term, the nuclear part is intertwined with the electron kinematics, i.e., the initial energy $\mathcal{E}$ and the energy transfer $y=\left(\mathcal{E}-\mathcal{E}^{\prime}\right) / \mathcal{E}$, but, fortunately, without a dependence upon $x_{\mathrm{Bj}}$. This favorable circumstance allows one to vary the kinematical variables upon which $g_{1 N}$ depends independently from the ones of the nuclear-structure $\mathcal{P}^{\hat{\mathrm{S}}_{A}}$, as discussed below.

For the sake of concreteness, let us consider how to extract information on the bound proton by using a ${ }^{3} \mathrm{He}$ target in pure spin state, polarized along the incident electron beam [remember that in the Bjorken limit it coincides with $\mathbf{q}$ and generalization to arbitrary polarization is straightforward; see Eqs. (45)-(49)]. This represents the longitudinally polarized target setting. The transversely polarized setting is quite important in the standard SiDIS, as briefly discussed in the following subsection and in more detail in Ref. [36]. For a reliable study of the structure function $g_{1 p}$ in the spectator SiDIS, with a detected deuteron, one has to minimize uncertainties induced by both the nuclear-structure terms and FSI effects. To this end, we fix the kinematics related to the spectral function, i.e., $\mathbf{p}_{\text {mis }}$, in such a way that FSI effects are minimized, while we vary $x_{N}$ (possibly taking $z_{N}$ constant). As clearly seen from Figs. 5-7, the most appropriate choice for this purpose is the parallel kinematics, $\theta_{\text {mis }}=180^{\circ}$ and $\left|\mathbf{p}_{\text {mis }}\right| \simeq 1 \mathrm{fm}^{-1}$. Under such conditions, one can safely discard the contribution containing either the perpendicular components of the spectral function $\mathcal{P}_{\perp}^{\hat{\mathbf{S}}_{A}}$ or terms proportional to $\left|\mathbf{p}_{N}\right|^{2} / 2 m_{N}^{2}=\left|\mathbf{p}_{\text {mis }}\right|^{2} / 2 m_{N}^{2} \ll 1$ (within our approximation, recall that $\mathbf{p}_{N}=-\mathbf{p}_{\text {mis }}$ ). Then, for $\mathbf{p}_{\text {mis }} \| \mathbf{k}_{e}$ one can approximate the term inside the brackets in Eq. (53) as follows:

$$
\begin{aligned}
{[\cdots] } & \approx \mathcal{P}_{\|}^{\frac{1}{2}}\left(\mathbf{p}_{\text {mis }}\right) \mathcal{E}\left[2-y-\frac{Q^{2}}{2 \mathcal{E}^{2}}+(2-y) \frac{\left|\mathbf{p}_{\text {mis }}\right|}{m_{N}}-\frac{\left|\mathbf{p}_{\text {mis }}\right|^{2}}{2 m_{N}^{2}}\right] \\
& \approx \mathcal{P}_{\|}^{\frac{1}{2}}\left(\mathbf{p}_{\text {mis }}\right) \mathcal{E}(2-y)\left[1+\frac{\left|\mathbf{p}_{\text {mis }}\right|}{m_{N}}\right] .
\end{aligned}
$$

where $\mathcal{P}_{\|}^{\frac{1}{2}}\left(\mathbf{p}_{\text {mis }}\right) \equiv \hat{\mathbf{p}}_{N} \cdot \mathcal{P}^{\frac{1}{2}}\left(\mathbf{p}_{\text {mis }}\right)$ and

$$
\begin{aligned}
\mathcal{K} \cdot \mathcal{P}^{\frac{1}{2}}\left(\mathbf{p}_{\text {mis }}\right) & =\left(\mathcal{E}+\mathcal{E}^{\prime} \cos \theta_{e}\right) \mathcal{P}_{\|}^{\frac{1}{2}}\left(\mathbf{p}_{\text {mis }}\right)+\mathcal{E}^{\prime} \sin \theta_{e} \mathcal{P}_{\perp}^{\frac{1}{2}}\left(\mathbf{p}_{\text {mis }}\right) \\
& =\sim\left[\mathcal{E}-\frac{Q^{2}}{2 \mathcal{E}}+\mathcal{E}^{\prime}\right] \mathcal{P}_{\|}^{\frac{1}{2}}\left(\mathbf{p}_{\text {mis }}\right) \\
& =\mathcal{E}\left[2-y-\frac{Q^{2}}{2 \mathcal{E}^{2}}\right] \mathcal{P}_{\|}^{\frac{1}{2}}\left(\mathbf{p}_{\text {mis }}\right) \\
\mathcal{K} \cdot \mathbf{p}_{N} & =-\left(\mathcal{E}+\mathcal{E}^{\prime} \cos \theta_{e}\right)\left|\mathbf{p}_{\text {mis }}\right| \\
& =-\mathcal{E}\left(2-y-\frac{Q^{2}}{2 \mathcal{E}^{2}}\right)\left|\mathbf{p}_{\text {mis }}\right|
\end{aligned}
$$

To have a "pure" factorized form of the corresponding expressions, let us define the reduced asymmetry in parallel 


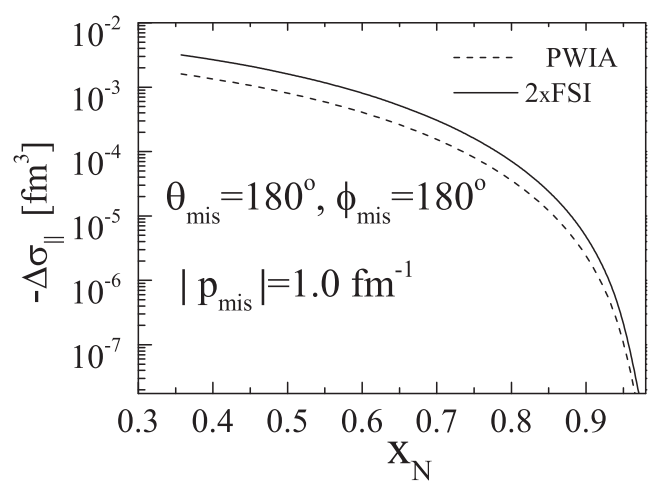

(a)

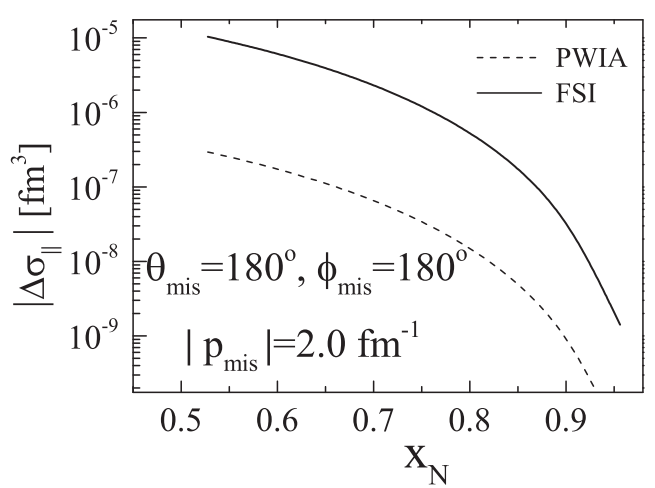

(b)

FIG. 8. The reduced parallel asymmetry [Eq. (56)] for the the reaction ${ }^{3} \mathrm{He}\left(\vec{e}, e^{\prime 2} \mathrm{H}\right) X$ vs $x_{N}$, with a longitudinally polarized target and choosing $\mathcal{E}=12 \mathrm{GeV}$ and $Q^{2}=6(\mathrm{GeV} / c)^{2}$. Dashed line, PWIA calculations; solid line, results with FSI effects. (Left) Calculations with $\left|\mathbf{p}_{\text {mis }}\right|=1 \mathrm{fm}^{-1}$, i.e., where FSI effects are negligible (cf. Fig. 5). (Right) The same as the left panel, but for $\left|\mathbf{p}_{\text {mis }}\right|=2 \mathrm{fm}{ }^{-1}$, i.e., where FSI effects are maximized and dominate the PWIA calculations by two orders of magnitude. Notice that in this panel the absolute value is shown, because the sign of the FSI calculation is negative (see text).

kinematics as follows:

$$
\begin{aligned}
\Delta \sigma_{\|} & \equiv \frac{\Delta \sigma^{\frac{1}{2}}\left(\theta_{\mathrm{mis}} \sim 180^{\circ}\right)}{d \varphi_{e} d x_{\mathrm{Bj}} d y d \mathbf{P}_{D}} /\left[4 \frac{(2-y) \alpha_{\mathrm{em}}^{2} m_{N}}{Q^{2} E_{N}}\right] \\
& =g_{1 N}\left(\frac{x_{B j}}{z_{N}}\right) \mathcal{P}_{\|}^{\frac{1}{2}}\left(\mathbf{p}_{\text {mis }}\right) .
\end{aligned}
$$

It is seen that the reduced asymmetry factorizes into two terms: one entirely determined by the structure function of the bound nucleon $g_{1 N}$, which in the present calculation is $g_{1 p}$, the second term being of a pure nuclear-structure origin. We reiterate that Eq. (56) has been obtained in the Bjorken limit and at low values of the missing momenta within the parallel kinematics.

In Fig. 8 numerical calculations of the reduced asymmetry (56) for the reaction ${ }^{3} \overrightarrow{\mathrm{He}}\left(\vec{e}, e^{\prime 2} \mathrm{H}\right) X$ are presented within the parallel kinematics at fixed values of $\left|\mathbf{p}_{\text {mis }}\right|$, as a function of $x_{N}=x_{\mathrm{Bj}} / z_{N}$, putting $\mathcal{E}=12 \mathrm{GeV}$ and $Q^{2}=6(\mathrm{GeV} / c)^{2}$. This setting allows one to determine (i) $p_{N}^{\mu} \equiv\left\{M_{\mathrm{He}}-\right.$ $\left.\sqrt{M_{D}^{2}+\left|\mathbf{p}_{\text {mis }}\right|^{2}}, \mathbf{p}_{\text {mis }}\right\}$, (ii) $v$ through $p_{N} q=Q^{2} /\left(2 x_{N}\right)$, and (iii) finally $x_{\mathrm{Bj}}$ and $z_{N}$. The left panel of Fig. 8 shows the parallel asymmetry at $\left|\mathbf{p}_{\text {mis }}\right|=1 \mathrm{fm}^{-1}$, i.e., in the case where the FSI corrections are small [cf. Fig. 5] and the study of the proton structure function, $g_{1 p}$ is highly feasible. It is very important to note that the calculations with FSI effects have been arbitrarily multiplied by a factor of two for making it distinguishable from the PWIA results. It should be pointed out that with such a kinematical choice, one has $\mathcal{P}_{\|}^{\mathrm{FSI}}\left(\mathbf{p}_{\text {mis }}\right)=$ $\mathcal{P}_{\|}^{\text {PWIA }}\left(\mathbf{p}_{\text {mis }}\right) \approx 1 \times 10^{-2} \mathrm{fm}^{3}$, and $0.27<x_{\mathrm{Bj}}<0.73$, so that $0.73<z_{N}<0.77$. The right panel illustrates the case at $\left|\mathbf{p}_{\text {mis }}\right|=2 \mathrm{fm}^{-1}$, where FSI effects are sizable: two orders of magnitude larger than the PWIA result. It is worth emphasizing that such a huge difference prevents a reliable extraction of $g_{1 p}$, at high missing momenta, even if the $\mathcal{P}_{\|}^{\text {PWIA }}$ and $\mathcal{P}_{\|}^{\text {FSI }}$ remain constant in the whole range of $x_{N}$ investigated. For $\left|\mathbf{p}_{\text {mis }}\right|=2 \mathrm{fm}^{-1}$, the scaling variable $x_{\mathrm{Bj}}$ varies in the interval $0.27<x_{\mathrm{Bj}}<0.48$ and $0.48<z_{N}<0.51$. As a final remark, we point out that, in our calculations, we have taken into account the difference between $v$ and $|\mathbf{q}|$, because in the adopted kinematics the Bjorken regime is not fully reached. This causes $z_{N}$ to slightly change with increasing $x_{\mathrm{Bj}}$, at fixed values of $\left|\mathbf{p}_{\text {mis }}\right|$ and $\theta_{\text {mis }}$.

We note in passing that, in Fig. 8, at $x \simeq 0.65$ one enters the resonance region and the present scheme has to be modified. The nucleon spin-dependent structure functions, as well as the effective cross section in the GEA approach, should be modified accordingly. For $x \geqslant 0.65$ the results shown in Fig. 8 are therefore not reliable. In the inclusive case, polarized electron scattering of ${ }^{3} \mathrm{He}$ in the resonance region has been discussed, e.g., in Refs. [44,54,55].

To tag the polarized EMC effect, i.e., if, how, and to what extent, the nucleon structure function in the medium differs from the free structure function, one has to get rid of the effects owing to the distorted nucleon spectral function and other nuclear-structure effects. One has to consider a quantity which would depend only upon $g_{1 N}\left(x_{\mathrm{Bj}}\right)$. This can be achieved by considering the ratio of parallel asymmetries [Eq. (56)], measured at two different values of the Bjorkenscaling variable $x_{\mathrm{Bj}}^{(1)}$ and $x_{\mathrm{Bj}}^{(2)}$, leaving unchanged all the other quantities. Therefore, exploiting the spectator SiDIS by a polarized ${ }^{3} \mathrm{He}$ in $2 \mathrm{bbu}$ channel, information on $g_{1 N}\left(x_{N}\right)$ can be obtained from the following ratio:

$$
R_{\|}\left(x_{\mathrm{Bj}}^{(1)}, x_{\mathrm{Bj}}^{(2)}\right)=\frac{\Delta \sigma_{\|}\left(x_{\mathrm{Bj}}^{(1)}, \mathbf{p}_{\mathrm{mis}}\right)}{\Delta \sigma_{\|}\left(x_{\mathrm{Bj}}^{(2)}, \mathbf{p}_{\mathrm{mis}}\right)}=\frac{g_{1 N}\left(x_{\mathrm{Bj}}^{(1)} / z_{N}^{(2)}\right)}{g_{1 N}\left(x_{\mathrm{Bj}}^{(2)} / z_{N}^{(2)}\right)} .
$$

In the Bjorken limit $z_{N}^{(1)}=z_{N}^{(2)}$, because $z_{N} \approx 1-\left|\mathbf{p}_{\text {mis }}\right| / m_{N}$ and therefore Eq. (57) directly reflects the $x_{N}$ dependence of $g_{1 N}$.

Let us now focus on the possibilities of using the spectator SiDIS to get information on the hadronization mechanism. As already discussed, for such purposes it is more convenient to consider the perpendicular kinematics, namely, when FSIs are dominant. The kinematics must be chosen in such a way that, by varying the missing momentum $\left|\mathbf{p}_{\text {mis }}\right|$, the argument of the nucleon structure function $g_{1 N}\left(x_{N}\right)$ remains constant. In this 


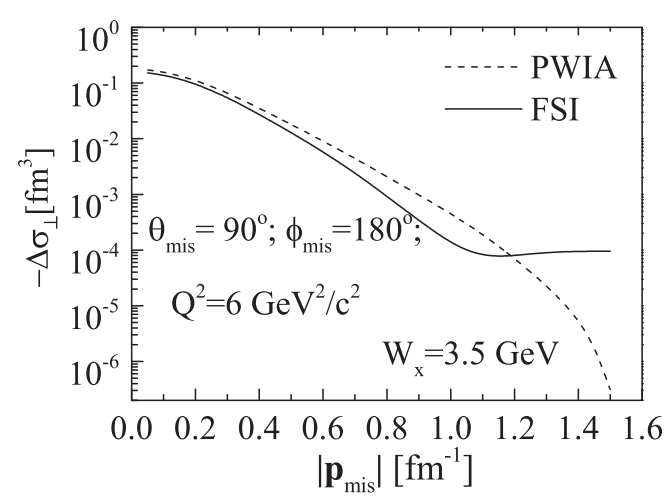

(a)

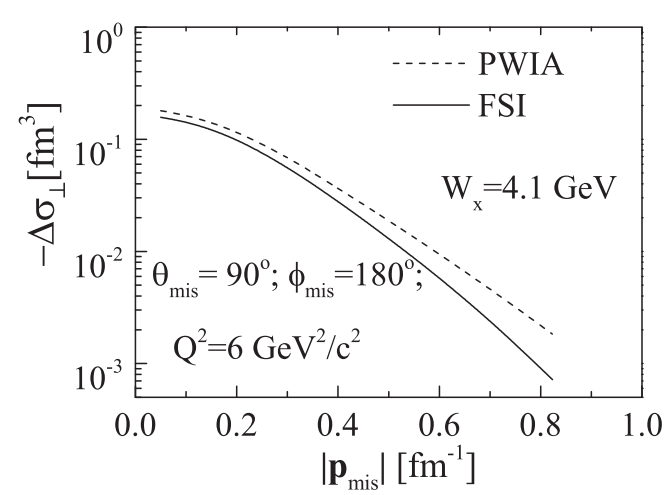

(b)

FIG. 9. The reduced perpendicular asymmetry [Eq. (59)] vs $\left|\mathbf{p}_{\text {mis }}\right|$ for the process ${ }^{3} \overrightarrow{\mathrm{He}}\left(\vec{e}, e^{\prime 2} \mathrm{H}\right) X$, with a longitudinally polarized target, choosing $\mathcal{E}=12 \mathrm{GeV}$ and $Q^{2}=6(\mathrm{GeV} / c)^{2}$, for two values of the invariant mass $W_{X}$. Dashed line, PWIA results; solid line, results with FSI taken into account. (Left) Calculations corresponding to $W_{X}=3.5 \mathrm{GeV}$, leading to $x_{N} \approx 0.34$. (Right) Calculations corresponding to $W_{X}=4.1 \mathrm{GeV}$, which yield $x_{N}=0.27$.

case the reduced asymmetry depends only on the spectralfunction components $\mathcal{P}_{\|}^{\frac{1}{2}}\left(\mathbf{p}_{\text {mis }}\right)$ and $\mathcal{P}_{\perp}^{\frac{1}{2}}\left(\mathbf{p}_{\text {mis }}\right)$.

In perpendicular kinematics, the term inside the square brackets in Eq. (53) becomes [remember that in perpendicular kinematics $\hat{\mathbf{p}}_{N} \cdot \mathcal{P}^{\hat{\mathbf{S}}_{A}}=\mathcal{P}_{\perp}^{\frac{1}{2}}\left(\mathbf{p}_{\text {mis }}\right)$ and moreover $\mathcal{K} \cdot \mathbf{p}_{N}=$ $\left.-\left|\mathbf{p}_{\text {mis }}\right| \mathcal{E}^{\prime} \sin \theta_{e}\right]$

$$
\begin{aligned}
{[\cdots]=} & \mathcal{P}_{\perp}^{\frac{1}{2}}\left(\mathbf{p}_{\text {mis }}\right)\left[\mathcal{E}^{\prime} \sin \theta_{e}+\frac{\left|\mathbf{p}_{\text {mis }}\right|}{m_{N}}\left(\mathcal{E}+\mathcal{E}^{\prime}\right)\right. \\
& \left.-\mathcal{E}^{\prime} \sin \theta_{e} \frac{\left|\mathbf{p}_{\text {mis }}\right|^{2}}{2 m_{N}^{2}}\right]+\mathcal{P}_{\|}^{\frac{1}{2}}\left(\mathbf{p}_{\text {mis }}\right)\left[\mathcal{E}+\mathcal{E}^{\prime} \cos \theta_{e}\right] \\
\approx & \mathcal{E}\left[\mathcal{P}_{\|}^{\frac{1}{2}}\left(\mathbf{p}_{\text {mis }}\right)\left(2-y-\frac{Q^{2}}{2 \mathcal{E}^{2}}\right)\right. \\
& \left.+\mathcal{P}_{\perp}^{\frac{1}{2}}\left(\mathbf{p}_{\text {mis }}\right)(2-y) \frac{\left|\mathbf{p}_{\text {mis }}\right|}{m}\right] \\
\approx & \mathcal{E}(2-y)\left[\mathcal{P}_{\|}^{\frac{1}{2}}\left(\mathbf{p}_{\text {mis }}\right)+\mathcal{P}_{\perp}^{\frac{1}{2}}\left(\mathbf{p}_{\text {mis }}\right) \frac{\left|\mathbf{p}_{\text {mis }}\right|}{m_{N}}\right],
\end{aligned}
$$

where also the term proportional to $\sin \theta_{e} \mathcal{E}^{\prime} / \mathcal{E}$ has been disregarded because in the DIS kinematics it becomes negligibly small. As in the parallel case, let us define the reduced perpendicular asymmetry

$$
\begin{aligned}
\Delta \sigma_{\perp} & \equiv \frac{\Delta \sigma^{\hat{\mathbf{s}}_{A}}\left(\theta_{\mathrm{mis}} \sim 90^{\circ}\right)}{d \varphi_{e} d x_{\mathrm{Bj}} d y d \mathbf{P}_{D}} /\left(\frac{2(2-y) \alpha_{\mathrm{em}}^{2} m_{N}}{Q^{2} E_{N}}\right) \\
& \approx g_{1 N}\left(\frac{x_{\mathrm{Bj}}}{z_{N}}\right)\left[\mathcal{P}_{\|}^{\frac{1}{2}}\left(\mathbf{p}_{\mathrm{mis}}\right)+\mathcal{P}_{\perp}^{\frac{1}{2}}\left(\mathbf{p}_{\mathrm{mis}}\right) \frac{\left|\mathbf{p}_{\mathrm{mis}}\right|}{m_{N}}\right] .
\end{aligned}
$$

In Fig. 9 the reduced asymmetry for a spectator SiDIS by a polarized ${ }^{3} \mathrm{He}$ target, with a detected deuteron, is presented as a function of the missing momentum in the perpendicular kinematics. In this case, one arranges the other kinematical variables in such a way that $x_{N}$ remains constant in the whole range of the explored $\left|\mathbf{p}_{\text {mis }}\right|$. In particular, once $\left|\mathbf{p}_{\text {mis }}\right|$ is given, then the four-momentum $p_{N}^{\mu}$ is determined (see above) and, after fixing both $Q^{2}$ and the invariant mass of the debris, $W_{X}^{2}=\left(p_{N}+q\right)^{2}$, one can straightforwardly determine
$x_{N}=Q^{2} /\left(W_{X}^{2}-p_{N}^{2}+Q^{2}\right)$ and, in turn, $y$. Because the considered $\left|\mathbf{p}_{\text {mis }}\right|$ is at most $1.5 \mathrm{fm}^{-1}$, one can immediately realize that $p_{N}^{2} \sim m_{N}^{2}$, and therefore $x_{N}$ is almost constant in the whole range of $\left|\mathbf{p}_{\text {mis }}\right|$. From Fig. 9, it is seen that FSI effects increase with $\left|\mathbf{p}_{\text {mis }}\right|$ and, for $\left|\mathbf{p}_{\text {mis }}\right|>0.6 \mathrm{fm}^{-1}$, become dominant. Therefore, by measuring the perpendicular asymmetry and comparing with the PWIA calculations one can get information on the magnitude of FSI. Because FSIs are determined by the effective debris-nucleon cross section, this should provide information on $\sigma_{\text {eff }}$ and, in turn, on the hadronization mechanism. Notice that the difference between $x_{\mathrm{Bj}}$ and $x_{N}$ is attributable to the off-mass-shellness of the hit nucleon, i.e., $p_{N}^{2} \neq m_{N}^{2}$, but at the considered values of $\left|\mathbf{p}_{\text {mis }}\right|$, this difference is negligibly small. Moreover, in the whole range of $\left|\mathbf{p}_{\text {mis }}\right|$ shown in Fig. 9, the value of $x_{N}$ remains almost constant. In the left panel, $x_{N} \approx 0.34$ and the proton structure function amounts to $g_{1 p}\left(x_{N}\right) \approx 0.174$.

In the right panel, the value of the invariant mass corresponds to an energy transfer close to the experimental kinematical limit $(v \approx 11.8 \mathrm{GeV})$ and it is reached when electrons scatter in the backward hemisphere. For this reason $\left|\mathbf{p}_{\text {mis }}\right|$ is kinematically constrained, with a cut $<1 \mathrm{fm}^{-1}$. Moreover, one has $x_{N}=0.273$ and $g_{1 p}\left(x_{N}\right) \approx 0.218$. As in the left panel, both PWIA and FSI nuclear-structure contributions are negative.

\section{B. Discussion}

The previous calculations, regardless of the direction of the polarization vector $\mathbf{S}_{A}$, show that all partial components of the spin-dependent spectral function [Eqs. (33), (34), and (51)] are different from zero, once FSI effects are taken into account. However, as expected, the components along $\mathbf{S}_{A}$ are much larger than the ones in the perpendicular direction [cf. Figs. 5 and 7]. When the asymmetries [Eq. (53)] are considered, one can see that the perpendicular component of the spectral function can appear only in scalar-product combination with $\mathbf{p}_{N}$. This leads to a further reduction, by a factor $\left|\mathbf{p}_{N}\right| / m_{N}$. Therefore, the perpendicular component of the spectral function can be safely neglected in the 
spectator SiDIS by a proper choice of kinematics. However, in general, the contribution of components perpendicular to the nuclear polarization vector cannot be a priori disregarded. In particular, if one considers a standard SiDIS reaction, i.e., with a fast hadron detected, and a transversely polarized ${ }^{3}$ He target, i.e., $\hat{\mathbf{S}}_{A} \perp \hat{\mathbf{k}}_{e} \sim \hat{\mathbf{q}}$, one should pay attention to FSI effects, because the contribution of the perpendicular components to the asymmetries can be, in principle, sizable. The above-mentioned SiDIS process is relevant for extracting the neutron transversity, but also Collins and Sivers functions, and therefore it has to be carefully investigated. This analysis will be presented elsewhere [36], but from the present study one can extract some general considerations and develop some expectations. In the transversely polarization setting of the target, the nuclear tensor contains contributions from both diagonal and non diagonal terms, viz. [cf. Eq. (46)]

$$
\begin{aligned}
W_{\mu \nu}^{\text {s.i. }}\left(S_{A \perp}, Q^{2}, P_{h}\right)= & \frac{1}{2} W_{\mu \nu}^{\frac{1}{2} \frac{1}{2}}+\frac{1}{2} W_{\mu \nu}^{-\frac{1}{2}-\frac{1}{2}} \\
& +\frac{1}{2}\left(W_{\mu \nu}^{\frac{1}{2}-\frac{1}{2}}+W_{\mu \nu}^{-\frac{1}{2} \frac{1}{2}}\right) .
\end{aligned}
$$

This entails that one has to calculate the whole polarization contribution to the spin-dependent spectral function, as given in Eqs. (34) and (51), to get information on FSI effects in standard SiDIS. Moreover, in standard SiDIS, the $(A-1)$ nucleus is not detected and one needs to integrate over all the values of the missing momentum; see Eq. (13). Indeed, because in the present paper we have evaluated the diagonal $\mathcal{P}_{\text {(FSI) }}^{\mathcal{M} M}$, one can infer that there should be kinematical ranges where FSI could modify the PWIA cross sections, but not necessarily the asymmetries. In particular, Fig. 5, where the diagonal $\left|\mathcal{P}_{\|}^{\frac{1}{2}}\left(\mathbf{p}_{\text {mis }}\right)\right|$ is shown for the parallel and perpendicular kinematics, indicates the kinematical region where one should expect large FSI effects [remember that the diagonal $\mathcal{P}_{\perp}^{\frac{1}{2}}\left(\mathbf{p}_{\text {mis }}\right)$ is always quite small]. Here we notice that, in principle, FSI effects on the cross section could be properly minimized if one measured a more exclusive SiDIS process, where both the $(A-1)$ spectator and the fast hadron are detected, i.e., by considering the reaction $l+\vec{A}=l^{\prime}+h+(A-1)+X$ with a fast hadron $h$ and a slow $(A-1)$ nucleus.

\section{CONCLUSIONS AND PERSPECTIVES}

In summary, we introduced a novel nuclear distribution function for investigating the SiDIS of polarized leptons by a polarized $A=3$ nucleus. Such a distribution function, the distorted spin-dependent spectral function, has been evaluated for an $A=3$ nucleus, by using the three-body wave function [49] corresponding to the realistic AV18 $N N$ interaction [28]. Our calculations should make more reliable the extraction of information on a bound nucleon, with a particular aim of providing a refined treatment of a polarized ${ }^{3} \mathrm{He}$ target, that, as is well-known, represents an effective neutron target, and plays a fundamental role for obtaining observables for the neutron like (i) transversity and (ii) Collins and Sivers functions, i.e., the main physical motivations of forthcoming experiments with a transversely polarized ${ }^{3} \mathrm{He}$ [17].
As a first step, we gave the formal expression of the nuclear tensor in terms of the nuclear overlaps, i.e., the transition amplitude between the target wave function and a state given by the Cartesian product of a nucleon plane wave and the fully interacting state composed by the remaining $(A-1)$ nucleons. Within such a formalism the nuclear tensor can be factorized in a nucleonic tensor and the proper combination of the above-mentioned overlaps, which in turn yield the well-known PWIA spin-dependent spectral function. Within the present approach, the relativistic effects are taken into account basically through the kinematics, postponing a fully Poincaré covariant approach, based on the light-front description of a Hamiltonian dynamical system, to a forthcoming paper (see Ref. [39] for a short presentation of the light-front spectral function). The FSI effects have been introduced through a GEA, successfully applied to the description of unpolarized spectator SiDIS processes [29]. The spectator SiDIS reactions, where a slowly recoiling $(A-1)$ system is detected in coincidence with the scattered lepton, differently from the standard SiDIS, where a fast hadron, produced after the virtual-photon absorption by a quark inside the bound nucleon, is detected, have been chosen as a testing ground for our approach. In particular, we have applied our formalism to the reaction ${ }^{3} \mathrm{He}\left(\vec{e}, e^{\prime 2} \mathrm{H}\right) X$, with a longitudinally polarized target. The investigation of the spin-dependent spectral function needed for describing the previous spectator SiDIS has made it possible to single out a kinematical region where the FSI can be minimized, and therefore one can safely extract the polarized structure function of a bound proton, $g_{1 p}$, and a region where the FSI has a maximal effect. There, one can address the issue of the hadronization in a nuclear medium. The analysis of this spectator SiDIS, though less complicate and less numerically demanding than the standard one, provides valuable information on a nucleon bound in a nucleus, shedding some light on the polarized EMC effect, and moreover allows us to calibrate our approach for the next application to the standard SiDIS by a transversely polarized ${ }^{3} \overrightarrow{\mathrm{He}}$. In this case we have to extended the calculation of the distorted spin-dependent spectral function to the off-diagonal terms, i.e., to the combination of the nuclear overlaps corresponding to target states with different spin projections, and then to integrate over a suitable (given by the experimental kinematics) range of missing momentum and energies of the $(A-1)$ system. Seemingly, this task appears highly nontrivial from the numerical point of view, while from the theoretical side the whole formalism can be derived following Eq. (48) and generalizing Eq. (43). Coming back to the spectator SiDIS, the approach presented in this paper and the numerical results for the spin-dependent spectral function (cf. Figs. 5-7) can be applied to a polarized tritium target, because for the time being we have disregarded the Coulomb effects. Through the reaction ${ }^{3} \overrightarrow{\mathrm{H}}\left(\vec{e}, e^{\prime 2} \mathrm{H}\right) X$ one could address the issue of the structure function of a bound neutron, $g_{1 n}$ and, analogously to the ${ }^{3} \mathrm{He}$ case, the hadronization process involving a quark inside a bound neutron. In spite of the apparent difficulties in getting a polarized ${ }^{3} \mathrm{H}$ target, one could conceive its construction as not impossible in a not too distant future, because in the past decade an impressive amount of research and development achievements has made available 
an unpolarized tritium target for planned and approved experiments [35].

\section{ACKNOWLEDGMENTS}

This work was supported by the Research Infrastructure Integrating Activity Study of Strongly Interacting Matter (acronym Hadron-Physics3, Grant Agreement No. 283286) under the Seventh Framework Programme of the European
Community and partially done under U.S. DOE Contract No. DE-AC05-06OR23177. Calculations were in part performed on Caspur facilities under the Standard HPC 2012, STD12-141 Grant "SRCnuc." We thank M. Alvioli for relevant support in running MPI codes. L.P.K. is grateful for the financial support from the JLab theory group and the program "Rientro dei Cervelli" of the Italian Ministry of University and Research. Moreover, L.P.K. acknowledges the warm hospitality of the JLab theory group and Patrizia Rossi for her very friendly support.
[1] J. Beringer et al. (Particle Data Group Collaboration), Phys. Rev. D 86, 010001 (2012).

[2] S. E. Kuhn, J.-P. Chen, and E. Leader, Prog. Part. Nucl. Phys. 63, 1 (2009).

[3] M. Arneodo, Phys. Rep. 240, 301 (1994).

[4] G. Piller and W. Weise, Phys. Rep. 330, 1 (2000).

[5] O. Hen, D. W. Higinbotham, G. A. Miller, E. Piasetzky, and L. B. Weinstein, Int. J. Mod. Phys. E 22, 1330017 (2013).

[6] J. J. Aubert et al. (European Muon Collaboration), Phys. Lett. B 123, 275 (1983).

[7] J. Ashman et al. (European Muon Collaboration), Phys. Lett. B 206, 364 (1988).

[8] V. Barone, A. Drago, and P. G. Ratcliffe, Phys. Rep. 359, 1 (2002).

[9] V. Barone, F. Bradamante, and A. Martin, Prog. Part. Nucl. Phys. 65, 267 (2010).

[10] U. D’Alesio and F. Murgia, Prog. Part. Nucl. Phys. 61, 394 (2007).

[11] J. L. Friar, B. F. Gibson, G. L. Payne, A. M. Bernstein, and T. E. Chupp, Phys. Rev. C 42, 2310 (1990).

[12] C. Ciofi degli Atti, S. Scopetta, E. Pace, and G. Salmè, Phys. Rev. C 48, R968 (1993).

[13] C. Ciofi degli Atti, E. Pace, and G. Salmè, Phys. Rev. C 46, R1591 (1992).

[14] R.-W. Schulze and P. U. Sauer, Phys. Rev. C 48, 38 (1993).

[15] C. Ciofi degli Atti, E. Pace, and G. Salmè, Phys. Rev. C 51, 1108 (1995).

[16] A. Kievsky, E. Pace, G. Salmè, and M. Viviani, Phys. Rev. C 56, 64 (1997).

[17] Cates G. et al., E12-09-018, JLAB approved experiment, hallaweb.jlab.org/collab/PAC/PAC38/E12-09-018-SIDIS.pdf;

X. Jiang et al., www.jlab.org/exp_prog/generated/apphalla.html; J. Arrington et al., www.jlab.org/ jinhuang/12GeV/ 12GeVLongitudinalHe3.pdf

[18] D. W. Sivers, Phys. Rev. D 41, 83 (1990); 43, 261 (1991).

[19] D. Boer and P. J. Mulders, Phys. Rev. D 57, 5780 (1998).

[20] J. C. Collins, Nucl. Phys. B 396, 161 (1993).

[21] S. J. Brodsky, D. S. Hwang, and I. Schmidt, Phys. Lett. B 530, 99 (2002).

[22] A. Airapetian et al. (HERMES Collaboration), Phys. Rev. Lett. 94, 012002 (2005).

[23] V. Y. Alexakhin et al. (COMPASS Collaboration), Phys. Rev. Lett. 94, 202002 (2005).

[24] S. J. Brodsky and S. Gardner, Phys. Lett. B 643, 22 (2006).

[25] X. Qian et al. (Jefferson Lab Hall A. Collaboration), Phys. Rev. Lett. 107, 072003 (2011).

[26] H. Gao et al., Eur. Phys. J. Plus 126, 2 (2011).
[27] S. Scopetta, Phys. Rev. D 75, 054005 (2007).

[28] R. B. Wiringa, V. G. J. Stoks, and R. Schiavilla, Phys. Rev. C 51, 38 (1995).

[29] C. Ciofi degli Atti and L. P. Kaptari, Phys. Rev. C 83, 044602 (2011).

[30] C. Ciofi degli Atti, L. P. Kaptari, and S. Scopetta, Eur. Phys. J. A 5, 191 (1999).

[31] C. Ciofi degli Atti, L. P. Kaptari, and B. Z. Kopeliovich, Eur. Phys. J. A 19, 145 (2004).

[32] S. Simula, Phys. Lett. B 387, 245 (1996).

[33] W. Melnitchouk, M. Sargsian, and M. I. Strikman, Z. Phys. A 359, 99 (1997).

[34] V. Palli, C. Ciofi degli Atti, L. P. Kaptari, C. B. Mezzetti, and M. Alvioli, Phys. Rev. C 80, 054610 (2009).

[35] B. D. Anderson et al., C12-10-103, JLAB approved experiment, hallaweb.jlab.org/collab/PAC/PAC37/C12-10-103-Tritium.pdf

[36] A. Del Dotto, L. P. Kaptari, E. Pace, G. Salmè, and S. Scopetta (unpublished).

[37] P. A. M. Dirac, Rev. Mod. Phys. 21, 392 (1949).

[38] B. D. Keister and W. N. Polyzou, Adv. Nucl. Phys. 20, 225 (1991).

[39] S. Scopetta, A. Del Dotto, E. Pace, and G. Salmè, Nuovo Cimento C 35, 101 (2012).

[40] C. Ciofi Degli Atti, Prog. Part. Nucl. Phys. 3, 163 (1980); H. Meier-Hajduk, C. Hajduk, P. U. Sauer, and W. Theis, Nucl. Phys. A 395, 332 (1983).

[41] S. Pollock, H. W. L. Naus, and J. H. Koch, Phys. Rev. C 53, 2304 (1996).

[42] S. A. Kulagin, W. Melnitchouk, G. Piller, and W. Weise, Phys. Rev. C 52, 932 (1995).

[43] S. A. Kulagin and R. Petti, Nucl. Phys. A 765, 126 (2006).

[44] S. A. Kulagin and W. Melnitchouk, Phys. Rev. C 78, 065203 (2008).

[45] J. A. O. Marinho, T. Frederico, E. Pace, G. Salmè, and P. U. Sauer, Phys. Rev. D 77, 116010 (2008).

[46] B. Bakamjian and L. H. Thomas, Phys. Rev. 92, 1300 (1953).

[47] L. P. Kaptari, K. Kazakov, A. Umnikov, and B. Kaempfer, Phys. Lett. B 321, 271 (1994); L. P. Kaptari, A. Yu. Umnikov, C. Ciofi degli Atti, S. Scopetta, and K. Yu. Kazakov, Phys. Rev. C 51, 52 (1995).

[48] U. Oelfke, P. U. Sauer, and F. Coester, Nucl. Phys. A 518, 593 (1990).

[49] A. Kievsky, M. Viviani, and S. Rosati, Nucl. Phys. A 577, 511 (1994).

[50] S. Kuhn, C. Keppel, and W. Melnitchouk (spokespersons), C. Ciofi degli Atti, L. Kaptari et al., www.jlab.org/exp_prog/proposals/03prop.html (PR03- 
012.pdf); http://www.jlab.org/exp_prog/proposals/06/PR12-06113.pdf

[51] L. L. Frankfurt, M. M. Sargsian, and M. I. Strikman, Phys. Rev. C 56, 1124 (1997).

[52] C. Ciofi degli Atti and L. P. Kaptari, Phys. Rev. Lett. 95, 052502 (2005).
[53] C. Ciofi degli Atti and L. P. Kaptari, Phys. Rev. Lett. 100, 122301 (2008).

[54] C. Ciofi degli Atti and S. Scopetta, Phys. Lett. B 404, 223 (1997).

[55] J. J. Ethier and W. Melnitchouk, Phys. Rev. C 88, 054001 (2013). 